\title{
Macrophage-derived VEGF-C decreases hematogenous metastatic dissemination by normalizing the tumor vasculature
}

Sabrina de Souza Ferreira ${ }^{1}$, Tatjana Wallmann ${ }^{1}$, Thomas Kerzel ${ }^{2}$, Majken Wallerius ${ }^{1}$, Margarita Bartish ${ }^{1}$, Laura-Sophie Landwehr ${ }^{1}$, Yangxun Pan ${ }^{1}$, Dennis Alexander Agardy ${ }^{1}$, Jonas Bergh ${ }^{1}$, Johan Hartman ${ }^{1,3}$, Mario Leonardo Squadrito ${ }^{2}$ and Charlotte Rolny ${ }^{1 \#}$.

${ }^{1}$ Karolinska Institute, Department of Oncology-Pathology, Stockholm, Sweden

${ }^{2}$ San Raffaele Telethon Institute for Gene Therapy (TIGET), Universitá Vita-Salute San Raffaele, Milan, Italy.

${ }^{3}$ Department of Clinical Pathology and Cytology, Karolinska University Laboratory, Stockholm, Sweden.

\#Corresponding author: Charlotte Rolny, Karolinska Institutet, Department of OncologyPathology, SciLifeLab, Solna 17176 Stockholm, Phone: +46(0)8-517 76 882, e-mail: charlotte.rolny@ki.se

Running Title: VEGF-C normalizes the tumor vasculature

Keywords: Vascular Endothelial Growth Factor-C, Tumor-Associated Macrophages, hypoxia, vascular normalization, metastatic dissemination

Conflicts of interest: JH obtained speaker's honoraria or advisory board remunerations from Roche, Novartis, AstraZeneca, Eli Lilly. None of the other authors have a conflict of interest to disclose.

Total number of words: 5874; Figures: 6 


\section{ABSTRACT}

Breast cancer $(\mathrm{BC})$ cells spread to secondary organs by exploiting lymphatic and blood vessels. Pro-angiogenic perivascular tumor-associated macrophages (TAMs) promote tumor cell intravasation and spreading to secondary organs by multiple mechanisms, including fueling tumor vessel abnormalization. Here, we show that perivascular TAMs expressing lymphangiogenic vascular endothelial growth factor (VEGF)-C normalize the tumor vasculature. This effect limited pulmonary metastases while enhancing lymph node metastasis. In contrast, tumor cell-derived VEGF-C promotes vascular abnormalization and pulmonary metastases. In human BC, expression of VEGF-C in TAMs inversely correlated with both malignant grade and occurrence of three-cell complexes composed of a perivascular macrophage, a tumor cell, and an endothelial cell (collectively defined as tumor microenvironment of metastasis, or TMEM), which are known to facilitate hematogenous cancer cell spreading. Conversely, tumor cellderived VEGF-C was associated with increased tumor grade and density of TMEM complexes. Our study reveals opposing effects of VEGF-C on BC metastasis that are dependent on the cellular source.

\section{INTRODUCTION}

$\mathrm{BC}$ is one of the most prevalent cancer worldwide (1). While the prognosis for nonmacrometastatic $B C$ is generally good due to efficient adjuvant therapies, including targeted and endocrine therapy, the prognosis for metastatic BC or triple-negative breast cancer (TNBC), is much worse partly due to resistance to current pharmacological therapies. New treatment strategies and biomarkers for these patients are therefore required.

Lymphangiogenic factors, including VEGF-C, promote tumor lymphangiogenesis via its receptor VEGFR3, facilitating lymphatic invasion (2) and tumor cell dissemination to the sentinel lymph nodes (3). The occurrence of tumor-draining lymph node metastasis is a prognostic indicator for systemic tumor spread in many types of cancers, including BC, and is linked to poor clinical outcomes (4). However, whether lymph node metastasis does in fact contribute to distant organ metastasis or only mirrors the general capacity of tumor cells to disseminate via the blood stream has been the subject of heavy debate in recent years (5). On one hand, experimental techniques such as cancer cell infusion into lymph nodes or tumor cell photoconversion indicated that a considerable proportion of lung metastases are indeed formed by lymph node-derived cancer cells $(6,7)$. However, by performing genetic analyses of clonal evolution between the primary tumor, lymph node metastases, and distant metastases in human breast cancer, we showed that tumor cells residing at distant sites stem from the 
primary tumor and not from the sentinel lymph nodes (8), suggesting that BC cells disseminate mainly via the hematogenous route to colonize distant organs.

Abnormal expression of VEGF-A results in atypical tumor vessels that are usually irregular, disorganized, permeable, and leaky, facilitating tumor cell intravasation into blood vessels, fueling distant metastasis (9). In contrast, normalization of the tumor vasculature is characteristically featured by enhanced vessel perfusion, reduced tumor hypoxia and increased pericyte coating of tumor vessels, resulting in hampering tumor cell intravasation (10). One major source of VEGF-A in tumors are TAMs. We and others, have shown that perivascular TAMs in experimental BC play a key role in regulating blood vessel integrity and the ability of cancer cells to invade blood vessels and spread to distant secondary organs $(11,12)$. Perivascular TAMs have in this context shown to play a major role in human BC metastasis as they form three-cell complexes together with a cancer cell expressing the invasive isoform of actin-binding protein mammalian enabled (MENA ${ }^{\text {inv }}$ ), and a CD31 ${ }^{+}$ endothelial cell, This complex is denoted as the tumor microenvironment of metastases (TMEMs), which has been shown to function as "doorway" for hematogenous dissemination both in experimental mammary tumor models (13) and clinically validated as a prognostic marker of metastasis in BC patients (14-16).

As TAMs dominate the immune landscape of $\mathrm{BC}$, we speculated that TAM-derived lymphangiogenic factors such as VEGF-C might regulate the route of metastatic dissemination. Thus, we hypothesized that the availability of lymphatic vessels in the primary tumor would promote lymphangiogenic spreading over hematogenous spreading. Unexpectedly, even though TAM-derived VEGF-C increased sentinel lymph node metastasis distant pulmonary metastases decreased indicating that enhanced lymph node metastasis does not fuel pulmonary seeding of tumor cells. The mechanism whereby TAM-derived VEGFC hampered pulmonary mechanism was ascribed to a phenotypic change of TAMs that augmented VEGFR3 expression on tumor vessels which facilitated tumor vessel normalization and functionality. Contrary, cancer cell-derived VEGF-C increased the occurrence of pulmonary metastasis and lymph node metastasis, which coincided with decreased expression of VEGFR3 on tumor vessels, which promoted their abnormalization. These data were substantiated in a cohort of $\mathrm{ER}^{+} \mathrm{BC}$ patients, showing that TAM-derived VEGF-C correlates inversely with $\mathrm{BC}$ malignant grade and the number of TMEM complexes, whereas the contrary was found for cancer cell-derived VEGF-C. In sum, we propose a mechanism whereby TAM-derived VEGF-C attenuates pulmonary metastasis via the increased VEGFR3 expression of tumor vessels, mediating vascular normalization and hampering tumor cell intravasation. 


\section{MATERIALS AND METHODS}

\section{Cell culture}

66cl4 and 4T1 tumor cells were grown in complete RPMI-1640 medium (Gibco) supplemented with $10 \%$ fetal bovine serum, $1 \%$ penicillin/streptomycin (Gibco)and $1 \%$ L-glutamine (Gibco). $4 \mathrm{~T} 1$ and $66 \mathrm{cl} 4$ mouse mammary carcinoma cells were originally derived from a single spontaneous tumor that arose in a $\mathrm{BALB} / \mathrm{cfC} 3 \mathrm{H}$ mouse and were purchased from the Karmanos Cancer Institute at Wayne State University.

\section{Bone-marrow derived macrophages (BMDMs)}

Bone marrow cells were acquired by flushing the femurs and tibias of female BALB/c mice. Erythrocytes were lysed with red blood cell lysis buffer (RCB, Sigma) and cells were cultured in RPMI-1640 medium (Gibco) supplemented with $10 \%$ fetal bovine serum, $1 \%$ penicillin/streptomycin (Gibco), 1 \% L-glutamine (Gibco) and $50 \mathrm{ng} / \mathrm{ml}$ M-CSF (Miltenyi) for 6to 7 days.

\section{Generation of VEGF-C vector}

VEGF-C ORF clone was purchased from Thermo Scientific Fisher and amplified by PCR (95 ${ }^{\circ} \mathrm{C}$ for $5 \mathrm{~min}, 5$ cycles at $95^{\circ} \mathrm{C}$ for $30 \mathrm{sec}, 45^{\circ} \mathrm{C}$ for $30 \mathrm{sec}, 72{ }^{\circ} \mathrm{C}$ for $30 \mathrm{sec}$ and 30 cycles at $95{ }^{\circ} \mathrm{C}$ for $30 \mathrm{sec}, 68^{\circ} \mathrm{C}$ for $30 \mathrm{sec}, 72^{\circ} \mathrm{C}$ for $30 \mathrm{sec}$ ). Primers for VEGF-C amplification are FW 5'-TAAAGACCGGTCAAAAGTTGCGAGCC-3' and RW 5'TCGGGGTCGACTACAATTTTCATTTTATTTTAAAC-3' primers. The DNA coding for VEGF$C$ was then inserted into a CMV.GFP.WPRE lentiviral vector sequence as described previously (17) downstream to the CMV promoter.

\section{Lentivirus production}

Vesicular stomatitis virus-G protein (VSV-G)-pseudotyped lentivirus vectors either empty or encoding VEGF-C were produced by transient five-plasmid co-transfection into HEK293T cells and concentrated using ultracentrifugation as described previously (18). Lentiviral vector titers were determined on HEK293T cells by TaqMan analysis as described previously (19). Lentiviral vectors were resuspended in PBS and stored at $-80^{\circ} \mathrm{C}$.

\section{Tumor experiments in mice}

Female BALB/c mice (4-6 weeks old) were purchased from Charles River Laboratory. $2 \times 10^{5}$ $4 \mathrm{~T} 1$ cells were injected into the mammary fat pad of anesthetized animals. Tumor size was measured externally using calipers, and tumor volumes were estimated using the following equation: $V_{\text {sphere }}=4 / 3 \pi \times(d / 2) 2 \times D / 2$ (d: minor tumor axis, $D$ : major tumor axis). Mice were 
euthanized 3- to 4 weeks after tumor cell injection, and tumors were weighed after dissection. All ethical permits were obtained from the Swedish Board of Agriculture (N65/16).

\section{Bone marrow transplant}

Bone marrow cells were acquired by flushing the femurs and tibias of 6- to 8-week-old female $\mathrm{BALB} / \mathrm{c}$ mice and treated with blood cell lysis buffer (Sigma). Lineage negative hematopoietic stem and progenitor cells (HSPCs) were purified using EasySep Isolation Kit (StemCell Technologies) according to manufacturer's instructions. $2 \times 10^{6}$ lineage negative cells $/ \mathrm{ml}$ were transduced with $1 \times 10^{8} \mathrm{TU} / \mathrm{ml}$ of empty vector or VEGF-C lentivirus in serum-free StemSpan medium (StemCell Technologies) supplemented with $10 \mathrm{ng} / \mathrm{ml}$ IL-3, $20 \mathrm{ng} / \mathrm{ml} \mathrm{IL-6,} 100 \mathrm{ng} / \mathrm{ml}$ SCF and $10 \mathrm{ng} / \mathrm{ml}$ FL-T3L (Stem Cell Technologies). $1 \times 10^{6}$ transduced HSPCs were resuspended in PBS and injected into the tail vein of 6-week-old female Balb/c mice that had undergone myeloablation using $20 \mathrm{mg} / \mathrm{kg}$ Busulfan (Sigma) by intraperitoneal (i.p.) injection on three consecutive days. Busulfan was solubilized in DMSO and diluted to $5 \mathrm{mg} / \mathrm{ml}$ in PBS. On the fourth day $12-14 \times 10^{6} \mathrm{BM}$ cells were given by tail vein injection. This procedure resulted in $80-98 \%$ chimerism (20).

\section{Metastasis colony assay}

Lungs and lymph nodes were dissected into an enzymatic buffer, dissociated to single cell suspensions and cultured in RPMI-1640 (complete medium) supplemented with $60 \mu \mathrm{M} 6-$ Thioguanine (Sigma) as previously described (21). Colonies formed by tumor cells resistant to 6-Thioguanine were fixed with $4 \%$ Formaldehyde (VWR Chemicals) and stained with Hematoxylin Harris (VWR Chemicals). Metastasis colonies were counted and the metastatic index was calculated by using the equitation $M_{\text {index }}=n / w$ ( $n$ : number of metastasis, w: weight of tumor) (21).

\section{Tumor hypoxia and vessel functionality}

Tumor hypoxia was assessed by intraperitoneal injection of $60 \mathrm{mg} / \mathrm{kg}$ pimonidazole hydrochloride (Hypoxiprobe) into tumor-bearing mice. Mice were sacrificed $1 \mathrm{~h}$ after injection and tumors were harvested. To detect the formation of hypoxic adducts, OCT tumor sections were immunostained with Hypoxyprobe-1-Mab-1 (Hypoxiprobe kit, Chemicon) following manufacturer's instructions. Vessel perfusion was evaluated by intravenous injection of $1 \mathrm{mg}$ of FITC-conjugated Lectin (Molecular Probes). Tumors were embedded in OCT material (VWR Chemicals) and snap-frozen in iso-pentane (VWR Chemicals) on dry ice.

\section{Tumor-bone-marrow-derived macrophages co-mingling assay.}


Bone-marrow derived macrophages (BMDM) were transduced with $1 \times 10^{8} \mathrm{TU} / \mathrm{ml}$ of empty vector or VEGF-C lentivirus vectors 4 days post-differentiation. These transduced BMDMs were then embedded in $200 \mu$ l of growth factor reduced Matrigel (BD Bioscience) together with $2 \times 10^{5} 4 \mathrm{~T} 1$ tumor cells, 2 days after transduction and injected subcutaneously into the flank of 8-week-old female BALB/c mice for 8 days.

\section{Immunofluorescence analysis}

Tumors embedded in OCT were cut in $10 \mu \mathrm{m}$ thick sections and fixed in methanol. Paraffinembedded tumor sections were treated for antigen retrieval with sodium citrate buffer (Thermo Scientific Fisher). All tumor sections were blocked in blocking buffer containing PBS (Sigma), $0.3 \%$ Triton X-100 (Sigma), $10 \%$ fetal bovine serum (Gibco) and $1 \%$ Bovine Serum Albumin (Sigma) and immunostained with the appropriate antibodies: anti CD68 (Dako), VEGF-A (SantaCruz), CD31 (BD Biosciences), Podoplanin (Life Technologies), Lyve-1 (Medical and Biological Laboratories), NG2 (Merck Millipore), a-SMA (Sigma-Aldrich), F4/80 (Bio-Rad), VEGF-C (SantaCruz), VEGFR3 (R\&D Systems), CD31 (DAKO), CD34 (DAKO), FITC-Lectin (Bio-Rad) and MENA/ENAH (Sigma). The appropriate secondary antibodies were conjugated with AlexaFluor 488, AlexaFluor 546, AlexaFluor 555 or AlexaFluor 647 fluorochromes (Life Technologies). Cell nuclei were labeled with DAPI (Invitrogen Corp). 10 independent fields from each section were analyzed by using LSM 710 Zeiss confocal microscope and quantified by ImageJ software.

\section{Patient material}

Paraffin-embedded whole tumor sections from breast cancer patients were obtained from Karolinska University Hospital and Southern Hospital, Stockholm, Sweden. All breast cancer patients at the Karolinska University Hospital and Southern Hospital have approved and signed a written consent for biobanking, including research purposes that have been approved by the local ethical committee (2016/957-31; ex vivo tumor models).

\section{Statistical analysis}

All data from representative experiments are presented with Standard Deviation (SD) and mean. Statistical analyses were performed using Student's t-test, two-way ANOVA or one-way ANOVA as indicated in the figure legends. Stars and hashtags in the graphs indicate significance as depicted in the figure legends. Differences were considered statistically significant at $p<0.05$. 


\section{RESULTS}

\section{VEGF-C is highly expressed in TAMs of $66 \mathrm{cl} 4$ tumors but not of $4 \mathrm{~T} 1$ tumors}

To elucidate the mechanism underlying the propensity of cancer cells to preferentially colonize lungs or lymph nodes, we employed the $4 \mathrm{~T} 1$ and $66 \mathrm{cl} 4$ murine $\mathrm{BC}$ cell lines which resemble human TNBCs (lackthe expression of estrogen (ER) and progesterone (PR) receptors, and the mouse homolog to the Human Epidermal Growth Factor Receptor 2, HER2, amplification). These two cell lines are metastatic variants of a single mouse mammary tumor (22). As previously shown by us (21) and others (22), 4T1 tumor cella preferentially disseminate to the lungs, while $66 \mathrm{cl} 4$ tumor cells predominantly metastasize to lymph nodes (Fig. 1A). Thus, we next investigated if the accumulation of lymphatic vessels differed between the two tumor models by using the blood endothelial marker CD31 and the lymphatic endothelial marker VEGFR3. Immunostaining for VEGFR3 and CD31 showed that VEGFR3 ${ }^{+}$CD $31^{-}$lymphatic vessels in the tumor periphery were more abundant in $66 \mathrm{cl} 4$ tumors as compared to 4T1 tumors (Fig. 1B,C). Unexpectedly, in the tumor center of the 66cl4 model, the larger part of the tumor vessels expressed VEGFR3 (Fig. 1D,E), as compared to the 4T1 model.

Lymphangiogenesis was further evaluated by using lymphatic endothelial cell markers LYVE1 and Podoplanin, which may also be expressed by a subset of TAMs $(23,24)$. Unexpectedly, the majority of 66cl4-infiltrating TAMs expressed LYVE1 (Fig. 1F,G) and Podoplanin (Supp. Fig. 1A,B), which was not observed for 4T1-infiltrating TAMs (Fig. 1F,G. and Supp. Fig. 1A,B). Thus, lymphatic vessel accumulation could not be further evaluated by using these markers. TAMs expressing Podoplanin (24) and/or LYVE1 (25) have been ascribed to be prolymphangiogenic and drive lymph node metastasis. We therefore further investigated if these observations correlated with enhanced levels of the pro-lymphangiogenic factor VEGF-C by 66cl4-infiltrating TAMs. Indeed, immunostaining showed that VEGF-C was more markedly expressed by 66cl4- as compared to 4T1-infiltrating TAMs (Fig. 1H,I). qPCR analysis of flow cytometry-based sorted F4/80'TAMs (Fig. 1J) further verified that 66cl4-derived TAMs express elevated levels of VEGF-C as compared to 4T1-derived TAMs. Flow cytometry sorted $\mathrm{GFP}^{+} 66 \mathrm{cl} 4$ and $\mathrm{GFP}^{+} 4 \mathrm{~T} 1$ cancer cells displayed similar levels of VEGF-C [but prominently lower than 66cl4 TAMs (Fig. $1 \mathrm{~J})$ ]. Further analysis revealed that the pro-angiogenic factor VEGF-A expression was markedly higher in 4T1-cancer cells and -infiltrating TAMs as compared to 66cl4-cancer cells and infiltrating TAMs (Supp. Fig. 1C). This was reflected by increased tumor vessel area in 4T1 tumors as compared to the 66cl4 tumors (Fig. Suppl. Fig 1D). The 4T1-tumor vessels also appeared to be more abnormal as they were less covered by neural/glial antigen 2 (NG2)-positive and/or a-smooth muscle actin ( $\alpha$-SMA)-positive 
pericytes as compared to the 66cl4 model (Suppl Fig 1E, F). Collectively, these data indicate that $66 \mathrm{cl} 4$-derived TAMs display elevated levels of VEGF-C, Podoplanin and LYVE1 coinciding with increased number of lymph node metastases and apparent normalized VEGFR3 ${ }^{+}$tumor vessels as compared to the $4 \mathrm{~T} 1$ tumor model.

\section{TAM-driven VEGF-C decreases pulmonary metastases}

It has previously been shown that VEGF-C expressing TAMs maintain VEGFR3 ${ }^{+}$vessel branching (26). We therefore hypothesized that expression of VEGF-C by TAMs could normalize tumor vessels and hamper pulmonary metastases. We therefore sought therefore to generate chimeric mice harboring haemopoietic cells, including TAMs, overexpressing VEGF-C. To this end, we transduced hematopoietic stem/progenitor cells (HSPCs) with lentiviral vectors (LVs) either empty or encoding VEGF-C, and transplanted the resulting cells into conditioned recipient mice (denoted CTR and VEGF-C chimeric mice, respectively) that had undergone myeloablation, achieved by busulfan treatment, affecting mainly bone marrowderived myeloid cells (20). 4T1 mammary cancer cells were then injected into the fat pad of the chimeric mice (Supp. Fig 2A). VEGF-C transduction measured by qPCR in bone marrow cells showed an average of 2 integrated lentiviral vector copies per mouse genome (Supp. Fig. 2B), corresponding to that $\sim 80 \%$ of all 4 T1-infiltrating TAMs expressed VEGF-C (Fig. 2A,B). Of note, we did not detect VEGF-C expression in immune cells apart from TAMs by using immunofluorescence for the pan-hemopoietic marker CD45 (Fig. 2A,B). VEGF-C protein expression in bone marrow-derived macrophages (BMDMs) obtained from transplanted CTR and VEGF-C-chimeric mice was further confirmed by western blot (Supp. Fig. 2C). Importantly, transduction of HSPCs with VEGF-C LVs did not affect VEGF-A expression in BMDMs (Supp. Fig. 2D).

Over-expression of VEGF-C in TAMs did not modify 4T1 tumor growth rate (Fig. 2C) or the final tumor burden (Supp. Fig. 2E), but increased lymph node metastases (metastatic index is measured by the number of lung colonies divided by tumor weight; Fig. 2D). Furthermore, TAM-derived VEGF-C reduced the pulmonary metastatic index by $40 \%$ compared to controls (Fig. 2E). 4T1-infiltrating VEGF-C ${ }^{+}$TAMs exhibited increased expression of LYVE1 (Fig. 2F,G) and Podoplanin (Supp. Fig 2F,G) compared to controls to a similar extent as was observed in 66cl4-infiltrating TAMs (Fig. 1F,G and Supp. Fig. 1A,B).

We next investigated if TAM-derived VEGF-C altered lymphangiogenesis and angiogenesis by performing immunofluorescence analysis of VEGFR3 and CD31 expression in tumor sections. As expected, the number of VEGFR3 ${ }^{+} \mathrm{CD} 31^{-}$lymphatic vessels in the tumor periphery was increased by TAM-derived VEGF-C as compared to 4T1 controls (Fig. $\mathbf{2 H}$ and I). As for 
the 66cl4 tumor model, where TAMs intrinsically express VEGF-C, enforced expression of VEGF-C in TAMs, elicited VEGFR3 expression on intratumoral CD31 ${ }^{+}$vessels as compared to 4 T1 controls (Fig. 2J and L). Furthermore, TAM-derived VEGF-C decreased tumor vessel area (Fig. 2K) whereas the number of blood vessels $/ \mathrm{mm}^{2}$ was increased (Supp. Fig. 2H) resulting in an apparent increased blood vessel density [\# of vessels divided by vessel area (Fig. 2M)], suggesting that TAM-derived VEGF-C promoted tumor blood vessel normalization. In sum, these data show that enforced expression of VEGF-C in TAMs leads to upregulation of LYVE1 and Podoplanin, mimicking the phenotype of 66cl4-infiltrating TAMs, which also coincided with decreased number of metastases in lungs, but increased in lymph nodes.

\section{TAM-driven VEGF-C normalizes the tumor vasculature}

Blood vessel normalization facilitates restriction of tumor cell invasion and pulmonary metastases $(10,11)$ and is associated with improved vessel perfusion, increased pericyte coverage as well as decreased tumor hypoxia $(10,27)$. FITC-conjugated lectin, which will only label functional blood vessels (i.e. with blood flow), and the hypoxic marker pimonidazole hydrochloride (PIMO) were injected intravenously and intraperitoneally respectively in 4T1tumor-bearing CTR and VEGF-C chimeric mice before euthanasia. Consistent with the increased vessel density above, 4T1 tumor sections from the VEGF-C chimeric mice displayed a significantly higher number of lectin perfused CD31 ${ }^{+}$vessels (Fig. 3A,B) and reduced tumor hypoxia (Fig. 3C,D) compared to controls. In line with these data, TAM-derived VEGF-C increased the number of blood vessels that were covered with $\alpha$-SMA and NG2 positive pericytes (Fig. 3E,F).

To further assess if VEGF-C expression leads TAMs to acquire an anti-metastatic function, we employed an in vivo tumor cell and BMDMs co-mingling assay (outlined in Suppl. Fig. 3A). In this assay, BMDMs are transduced with either CTR or VEGF-C LVs, mixed in matrigel with 4T1 cancer cells and injected subcutaneously into syngeneic mice. Implants were harvested 8 days post-injection (before the onset of the humoral immune system). Immunostaining revealed that VEGF-C-engineered BMDMs attenuated VEGFR3 expression on blood vessels (Fig. 4A,B) to similar levels as was observed for VEGF-C expressing TAMs (Fig. 2J and L). Further immunofluorescence analysis showed that perfusion and vessel normalization was augmented by TAM-derived VEGF-C as indicated by an increased number of vessels labeled with FITC-conjugated lectin (Fig. 4C,D), whereas vessel permeability (Suppl. Fig. 3B,C) and hypoxic areas were reduced as compared to controls (Fig. 4E,F). Also, TAM-derived VEGF-C augmented $\mathrm{NG}_{2}{ }^{+}$and $\alpha-\mathrm{SMA}^{+}$pericyte coating of tumor vessels as compared to controls (Fig. 4G,H). As we cannot study distant metastases in this particular tumor model, we evaluated the occurrence of TMEM complexes, which were decreased in tumors that received VEGF C- 
expressing BMDMs as compared to controls (Fig. 4I,J). In summary, VEGF-C-expressing TAMs increased vessel normalization as well as function in tumors, and both were associated with decreased occurrence of TMEM complexes.

\section{TAM-derived VEGF-C does not alter the tumor immune composition}

Lymphatic vessels are extensively involved in the regulation of immune cell trafficking to the tumor, which may affect tumor growth and metastatic dissemination (5). We therefore investigated if TAM-derived VEGF-C could affect the intratumoral immune composition by employing flow cytometry analysis. VEGF-C overexpression in TAMs did not affect accumulation of total CD11 $\mathrm{b}^{+}$myeloid cells (Supp. Fig. 4A), CD11 b ${ }^{+} \mathrm{F} 4 / 80^{+}$TAMs (Suppl. Fig. 4B), Ly6G ${ }^{+}$neutrophils (Suppl. Fig. 4C), Ly6C ${ }^{+}$monocytes (Suppl. Fig. 4D), immunoactivating [also referred to M1-like TAMs (Suppl Fig. 4E)] or immunosuppressive TAMs [also known as M2-like TAMs (Suppl. Fig. 4F)]. Immunoactivating TAMs were identified by high expression

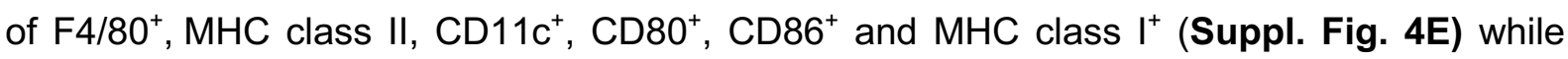
immunosuppressive $\mathrm{F} 4 / 80^{+}$TAMs were identified by low expression of MHC class II or elevated levels of mannose receptor c-type 1 (MRC1) (Suppl. Fig. 4F). Of note, BMDMs engineered to express VEGF-C did not display altered expression levels of immunostimulatory cytokines such as C-X-C motif ligand (CxCl) 9, 10, 11, interleukin (IL) 1a, or immunosuppressive factors including II10, arginase1 (Arg1), Mrc1 and transforming growth factor (Tgf)- $\beta$, detected by qPCR (Suppl. Fig. 4G). This indicates that VEGF-C does not alter the activation or the polarization status of macrophages. Finally, TAM-driven VEGF-C did not affect the accumulation of either $\mathrm{CD} 4^{+} \mathrm{T}$ cells, $\mathrm{CD} 8^{+} \mathrm{T}$ cells or $\mathrm{CD} 49 \mathrm{~b}^{+} \mathrm{NK}$ cells (Suppl. Fig. 4H). Hence, these data show that enforced expression of VEGF-C in HPSCs does not affect the intratumoral immune composition or the TAM phenotype.

\section{Perivascular TAM-derived VEGF-C inversely correlates to the number of TMEM complexes and grade of malignancy in human breast cancer}

Accumulation of TAMs has been linked to poor clinical outcomes in several cancers, including BC (28). Based on our experimental data, we speculated that perivascular TAM expressing VEGF-C might be of an angiostatic phenotype and, therefore, be more frequent in benign $\mathrm{BC}$ as compared to malign BC. The majority of TNBC patient specimens that are available are of high-grade, therefore we focused on estrogen receptor (ER)-positive breast cancer, which is also the most prevalent type in $\mathrm{BC}$ patients $(\sim 70 \%)$ that are candidates for endocrine therapy. However, half of the treated patients will develop metastatic disease within 20-years of diagnosis (29). Hence, we evaluated the accumulation of bulk and perivascular TAMs by immunostaining of tumor sections obtained from $\mathrm{ER}^{+}$ductal $\mathrm{BC}$ patient specimens of different histological grades (Nottingham Histological Grade I-III, where grade I and II indicates low 
grade, and grade III accounts for high grade). CD68 ${ }^{+}$TAMs (Fig. 5A,B) and perivascular TAMs (Fig. 5A,C) accumulated at a higher extent in high-grade than in low-grade $\mathrm{ER}^{+} \mathrm{BC}$. Additional immunofluorescence staining, which also included VEGF-C, revealed that perivascular TAMs expressed VEGF-C significantly higher in low-grade than in high-grade $\mathrm{ER}^{+} \mathrm{BC}$ (Fig. 5D,E), while the contrary was found for cancer cells [stained for MENA, not shown (Fig. 5F,G)].

As we lack information for distant metastases for this patient cohort, we evaluated the occurrence of TMEM complexes, a clinically validated prognostic marker for the occurrence of distant metastasis in BC patients (14,30,31). Immunofluorescence staining of TMEM complexes revealed that the number of TMEM complexes was significantly higher in highgrade than in low-grade $\mathrm{ER}^{+} \mathrm{BC}$ tumors (Fig. $\mathbf{5 H}, \mathrm{I}$ ). Importantly, accumulation of TMEM complexes (normalized to the number of $\mathrm{CD} 31^{+}$vessels, as the number of vessels was not affected by the grade of malignancy; Suppl. Fig. 5A) correlated inversely with the presence of perivascular TAMs expressing VEGF-C (Fig. 5J). Instead, cancer cell-derived VEGF-C correlated with an increased number of TMEMs (Fig. 5K). In sum, these data indicate that the presence of perivascular TAMs expressing VEGF-C correlates inversely with the presence of TMEM complexes that enable hematogenic tumor cell dissemination in human BC. The opposite was found for VEGF C-expressing tumor cells.

\section{Cancer cell-derived VEGF-C enhances pulmonary and lymph node metastases, accompanied by a pro-metastatic tumor microenvironment}

We next evaluated the effect of cancer cell-derived VEGF-C in the 4T1 mammary tumor mouse model. Hence, 4T1 cancer cells were transduced with empty or VEGF C-expressing LVs to originate 4T1-CTR and 4T1-VEGF-C cancer cells, which then were inoculated in the mammary fat-pad of syngeneic mice. Cancer cell-derived VEGF-C did not alter tumor growth or burden compared to controls (Fig. 6A and Suppl. Fig 6A) but increased lymph node (Fig. 6B) and pulmonary metastasis (Fig. 6C). The higher number of proximal and distal metastases was associated with an increased number of peri-tumoral VEGFR3 ${ }^{+}$lymphatic vessels (Fig. 6D,E) and tumor hypoxia (Fig. 6F,G) while vessel perfusion (Fig. 6H,I) and pericyte coverage of tumor blood vessels were decreased (Fig. 6J,K) as compared to controls. Furthermore, cancer cell-derived VEGF-C reduced expression of VEGFR3 on blood-tumor vessels as compared to controls (Fig. 6M,N). In sum, cancer cell-derived VEGF-C augmented proximal and distant metastases, concomitant with a pro-metastatic tumor microenvironment.

\section{DISCUSSION}

TAMs are recognized as regulators of tumor progression and have been linked to poor clinical outcomes in most cancers (9-11). Like macrophages in many other tissues, TAMs show 
remarkable functional plasticity and often exhibit a spectrum of activation states that span from an anti-tumoral, pro-inflammatory and angiostatic phenotype (sometimes referred to as M1like) to a pro-tumoral, immunosuppressive and angiogenic (often referred to as M2-like) phenotype (32). Usually, TAMs will express markers characteristic of both activation states and their function will partly depend on their spatial localization (33). For instance, perivascular macrophages, including TIE2 ${ }^{+}$TAMs, promote angiogenesis (34) by secreting factors such as VEGF-A facilitating vessel permeability and cancer cell intravasation $(13,15)$. Perivascular TAMs are furthermore a part of the TMEM three-cell complex together with MENA $A^{\text {inv }}$ expressing cancer cells and endothelial cells that function as gateways to hematogenous spreading (15). In our study, we showed that the occurrence of TMEM complexes correlated to grade of malignancy and cancer cell-derived VEGF-C expression but inversely to the accumulation of VEGF-C-expressing perivascular TAMs.

VEGF-C stimulates lymphangiogenesis via activation of VEGFR3 but can also regulate angiogenesis by binding to VEGFR2/VEGFR3 heterodimers, expressed by blood vessels during embryogenesis, endothelial tip cells in adults and tumor blood vessels $(26,35)$. In this respect, TAM derived VEGF-C has been reported to maintain the maturation of blood vessels that express VEGFR3 (26). In contrast, deletion of VEGFR3 in blood vessels enhances VEGFR2 expression that stimulates the VEGFA/VEGFR2 pathway, resulting in excessive vascular permeability (36) and hypersprouting (26). and hyperplasia upon encountering a VEGF-C expressing macrophage (26). Consistently, in our model. perivascular VEGF$\mathrm{C}^{+}$TAMs, increased VEGFR3 expression on tumor vessels, resulting in augmentation of vessel normalization and hampering of pulmonary metastasis. In contrast, cancer cell-derived VEGF$C$ reduced VEGFR3 expression on blood tumor vessels, leading to increased vessel abnormalization coinciding with increased pulmonary metastasis formation. We hypothesize that perivascular VEGF-C ${ }^{+}$TAMs stimulate endothelial cells to express VEGFR3, in a localdose dependent fashion, facilitating tumor vessel normalization. In contrast, tumor-derived VEGF-C will most likely be distributed throughout the tumor, failing to create a specific local source of VEGF-C. Of note, VEGF-C expression by TAMs did not alter their expression of proinflammatory or immunosuppressive cytokines nor did it effect the autocrine levels of VEGFA. Finally, both TAM-derived or cancer cell-derived VEGF-C increased the occurrence of lymph node metastases, even though the latter were much more pronounced. Collectively, our data show that TAM-derived VEGF-C plays an unexpected role in normalizing the tumor vasculature and decreasing hematogenous cancer cell spreading. Further clinical relevance for VEGF-C expressing TAMs as potential biomarkers were established in a cohort of ER+ $\mathrm{BC}$ specimens as these TAMs correlated inversely with malignant grade and the occurrence of TMEM complexes. Importantly the opposite was fond for cancer cell-derived VEGF-C. In 
sum our data suggest relevance of VEGF-C-expressing TAMs in regulating hematogenous cancer cell spreading.

\section{FINANCIAL SUPPORT}

This study was supported by the Swedish Cancer Society (2016/825 and 2019/0281), Swedish Scientific Council (2018-5982), Radiumhemmets research funds (154352) to CR; Swedish Cancer Society and the Wallenberg foundation to JB; TW was supported by KI-PhD (20142018) foundation. JH was supported by grants from Radiumhemmets research funds, Swedish Breast Cancer Association, Swedish Society for Medical Research. MLS was supported by Fondazione Regionale per la Ricerca Biomedica (FRRB) 1751658-2020 and CARIPLO 18342019.

All the authors declare no conflict of interest regarding the work that is being submitted.

\section{REFERENCES}

1. Waks AG, Winer EP. Breast Cancer Treatment: A Review. JAMA 2019;321:288-300

2. Tacconi C, Correale C, Gandelli A, Spinelli A, Dejana E, D'Alessio S, et al. Vascular endothelial growth factor $\mathrm{C}$ disrupts the endothelial lymphatic barrier to promote colorectal cancer invasion. Gastroenterology 2015;148:1438-51 e8

3. Dieterich LC, Detmar M. Tumor lymphangiogenesis and new drug development. Adv Drug Deliv Rev 2016;99:148-60

4. Jana S, Muscarella RA, Jr., Jones D. The Multifaceted Effects of Breast Cancer on Tumor-Draining Lymph Nodes. Am J Pathol 2021;191:1353-63

5. Petrova TV, Koh GY. Biological functions of lymphatic vessels. Science 2020;369

6. Brown M, Assen FP, Leithner A, Abe J, Schachner H, Asfour G, et al. Lymph node blood vessels provide exit routes for metastatic tumor cell dissemination in mice. Science 2018;359:1408-11

7. Pereira ER, Kedrin D, Seano G, Gautier O, Meijer EFJ, Jones D, et al. Lymph node metastases can invade local blood vessels, exit the node, and colonize distant organs in mice. Science 2018;359:1403-7

8. Ullah I, Karthik GM, Alkodsi A, Kjallquist U, Stalhammar G, Lovrot J, et al. Evolutionary history of metastatic breast cancer reveals minimal seeding from axillary lymph nodes. $\mathrm{J}$ Clin Invest 2018;128:1355-70

9. Goel S, Wong AH, Jain RK. Vascular normalization as a therapeutic strategy for malignant and nonmalignant disease. Cold Spring Harb Perspect Med 2012;2:a006486

10. Mazzone M, Dettori D, Leite de Oliveira R, Loges S, Schmidt T, Jonckx B, et al. Heterozygous deficiency of PHD2 restores tumor oxygenation and inhibits metastasis via endothelial normalization. Cell 2009;136:839-51

11. Rolny C, Mazzone M, Tugues S, Laoui D, Johansson I, Coulon C, et al. HRG inhibits tumor growth and metastasis by inducing macrophage polarization and vessel normalization through downregulation of PIGF. Cancer Cell 2011;19:31-44

12. Mazzieri R, Pucci F, Moi D, Zonari E, Ranghetti A, Berti A, et al. Targeting the ANG2/TIE2 axis inhibits tumor growth and metastasis by impairing angiogenesis and disabling rebounds of proangiogenic myeloid cells. Cancer Cell 2011;19:512-26

13. Harney AS, Arwert EN, Entenberg D, Wang Y, Guo P, Qian BZ, et al. Real-Time Imaging Reveals Local, Transient Vascular Permeability, and Tumor Cell Intravasation Stimulated by TIE2hi Macrophage-Derived VEGFA. Cancer Discov 2015;5:932-43 
14. Rohan TE, Xue X, Lin HM, D'Alfonso TM, Ginter PS, Oktay MH, et al. Tumor microenvironment of metastasis and risk of distant metastasis of breast cancer. J Natl Cancer Inst 2014;106

15. Karagiannis GS, Pastoriza JM, Wang Y, Harney AS, Entenberg D, Pignatelli J, et al. Neoadjuvant chemotherapy induces breast cancer metastasis through a TMEM-mediated mechanism. Sci Transl Med 2017;9

16. Ginter PS, Karagiannis GS, Entenberg D, Lin Y, Condeelis J, Jones JG, et al. Tumor Microenvironment of Metastasis (TMEM) Doorways Are Restricted to the Blood Vessel Endothelium in Both Primary Breast Cancers and Their Lymph Node Metastases. Cancers (Basel) 2019;11

17. Keklikoglou I, Cianciaruso C, Guc E, Squadrito ML, Spring LM, Tazzyman S, et al. Chemotherapy elicits pro-metastatic extracellular vesicles in breast cancer models. Nat Cell Biol 2019;21:190-202

18. De Palma M, Naldini L. Transduction of a gene expression cassette using advanced generation lentiviral vectors. Methods Enzymol 2002;346:514-29

19. De Palma M, Naldini L. Transduction of a gene expression cassette using advanced generation lentiviral vectors. Methods in enzymology 2002;346:514-29

20. Lund H, Pieber M, Parsa R, Han J, Grommisch D, Ewing E, et al. Competitive repopulation of an empty microglial niche yields functionally distinct subsets of microglia-like cells. Nat Commun 2018;9:4845

21. Casazza A, Fu X, Johansson I, Capparuccia L, Andersson F, Giustacchini A, et al. Systemic and targeted delivery of semaphorin $3 \mathrm{~A}$ inhibits tumor angiogenesis and progression in mouse tumor models. Arterioscler Thromb Vasc Biol 2011;31:741-9

22. Aslakson CJ, Miller FR. Selective events in the metastatic process defined by analysis of the sequential dissemination of subpopulations of a mouse mammary tumor. Cancer Res 1992;52:1399-405

23. Pucci F, Venneri MA, Biziato D, Nonis A, Moi D, Sica A, et al. A distinguishing gene signature shared by tumor-infiltrating Tie2-expressing monocytes (TEMs), blood "resident" monocytes and embryonic macrophages suggests common functions and developmental relationships. Blood 2009

24. Bieniasz-Krzywiec P, Martin-Perez R, Ehling M, Garcia-Caballero M, Pinioti S, Pretto $\mathrm{S}$, et al. Podoplanin-Expressing Macrophages Promote Lymphangiogenesis and Lymphoinvasion in Breast Cancer. Cell Metab 2019;30:917-36 e10

25. Bron S, Henry L, Faes-Van't Hull E, Turrini R, Vanhecke D, Guex N, et al. TIE-2expressing monocytes are lymphangiogenic and associate specifically with lymphatics of human breast cancer. Oncoimmunology 2016;5:e1073882

26. Tammela T, Zarkada G, Nurmi H, Jakobsson L, Heinolainen K, Tvorogov D, et al. VEGFR-3 controls tip to stalk conversion at vessel fusion sites by reinforcing Notch signalling. Nat Cell Biol 2011;13:1202-13

27. Rankin EB, Giaccia AJ. Hypoxic control of metastasis. Science 2016;352:175-80

28. Zhao X, Qu J, Sun Y, Wang J, Liu X, Wang F, et al. Prognostic significance of tumorassociated macrophages in breast cancer: a meta-analysis of the literature. Oncotarget 2017;8:30576-86

29. Sheppard VB, Faul LA, Luta G, Clapp JD, Yung RL, Wang JH, et al. Frailty and adherence to adjuvant hormonal therapy in older women with breast cancer: CALGB protocol 369901. J Clin Oncol 2014;32:2318-27

30. Oktay MH, Gertler FB, Liu YF, Rohan TE, Condeelis JS, Jones JG. Correlated immunohistochemical and cytological assays for the prediction of hematogenous dissemination of breast cancer. J Histochem Cytochem 2012;60:168-73

31. Robinson BD, Sica GL, Liu YF, Rohan TE, Gertler FB, Condeelis JS, et al. Tumor microenvironment of metastasis in human breast carcinoma: a potential prognostic marker linked to hematogenous dissemination. Clin Cancer Res 2009;15:2433-41

32. Mantovani A, Marchesi F, Malesci A, Laghi L, Allavena P. Tumour-associated macrophages as treatment targets in oncology. Nat Rev Clin Oncol 2017;14:399-416 
33. Yang M, McKay D, Pollard JW, Lewis CE. Diverse Functions of Macrophages in Different Tumor Microenvironments. Cancer Res 2018;78:5492-503

34. De Palma M, Murdoch C, Venneri MA, Naldini L, Lewis CE. Tie2-expressing monocytes: regulation of tumor angiogenesis and therapeutic implications. Trends Immunol 2007;28:519-24

35. Roy H, Bhardwaj S, Yla-Herttuala S. Biology of vascular endothelial growth factors. FEBS Lett 2006;580:2879-87

36. Heinolainen K, Karaman S, D'Amico G, Tammela T, Sormunen R, Eklund L, et al. VEGFR3 Modulates Vascular Permeability by Controlling VEGF/VEGFR2 Signaling. Circ Res 2017; 120:1414-25 
Figure 1 preprint (which was not certified by peer review) is the author/funder. All rights reserved. No reuse allowed without permission.

A.

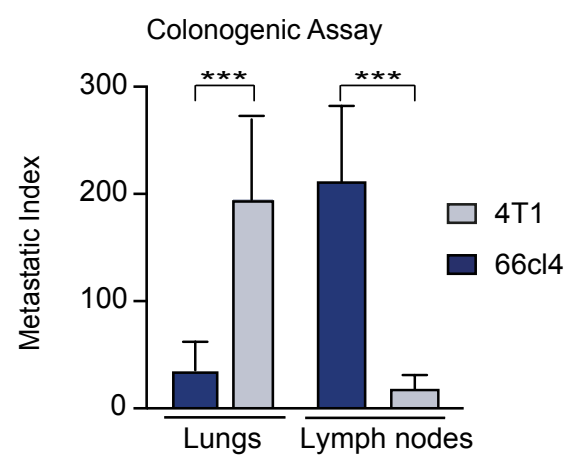

D.

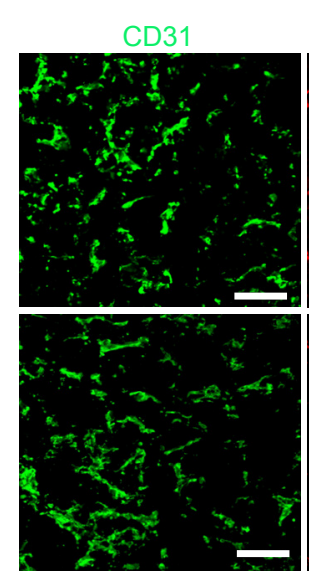

Tumor center

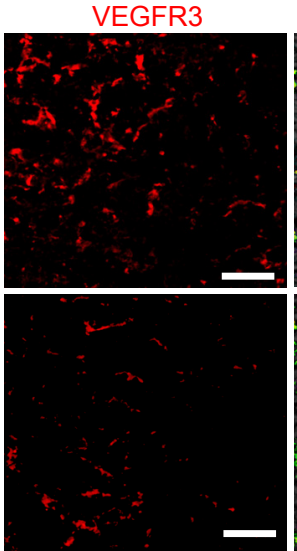

F.

CD31

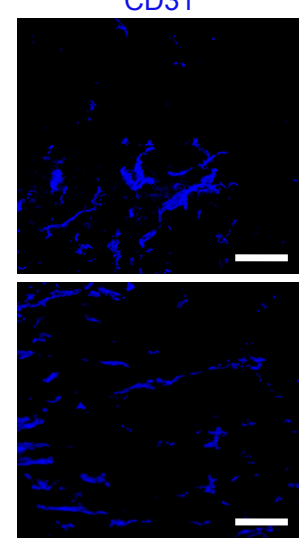

H.

F4/80

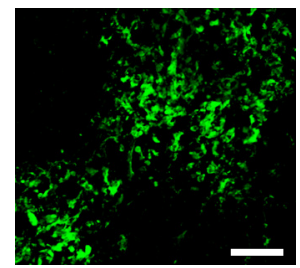

4T1
B.

$66 \mathrm{cl} 4$

4T1

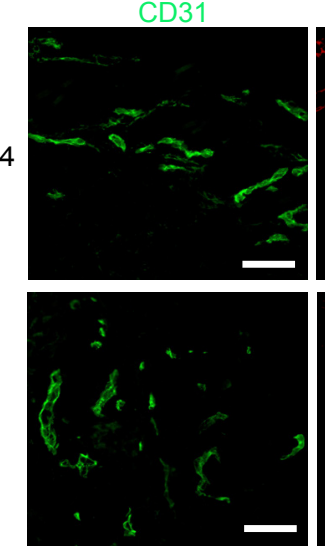

Tumor periphery VEGFR3

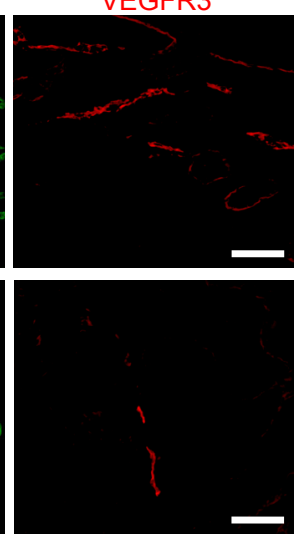

C.

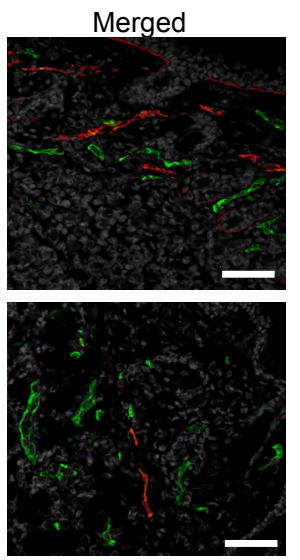

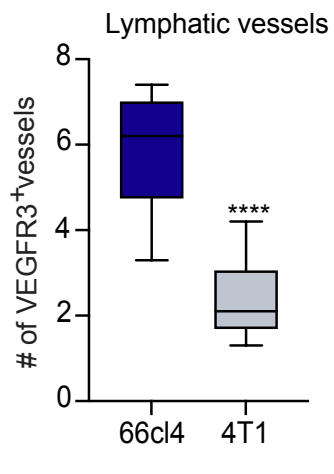

E. VEGFR3 ${ }^{+} \mathrm{CD} 31^{+}$blood vessels
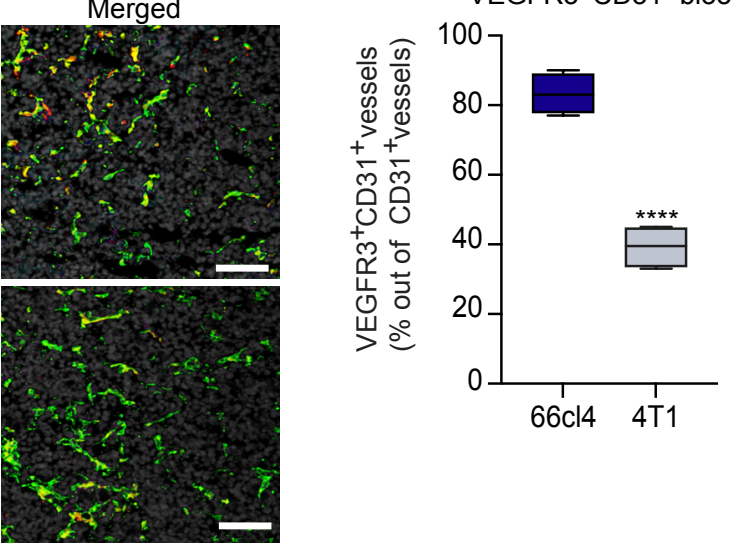

G.
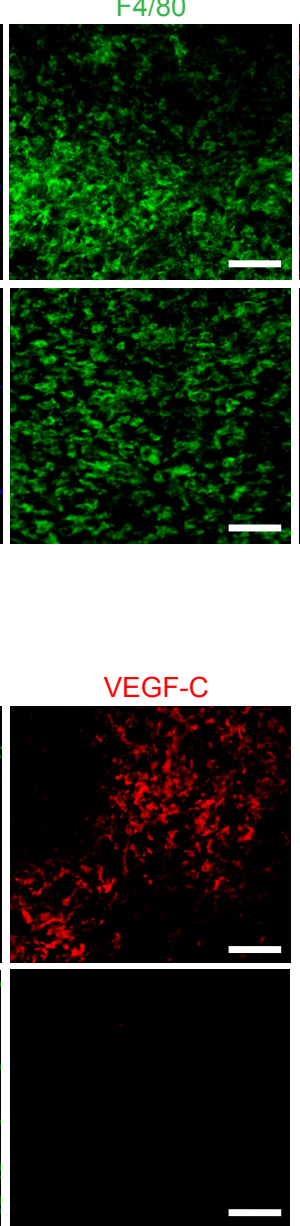

LYVE-1

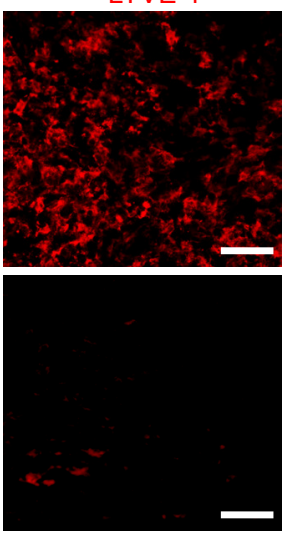

I.

Merged

Merged
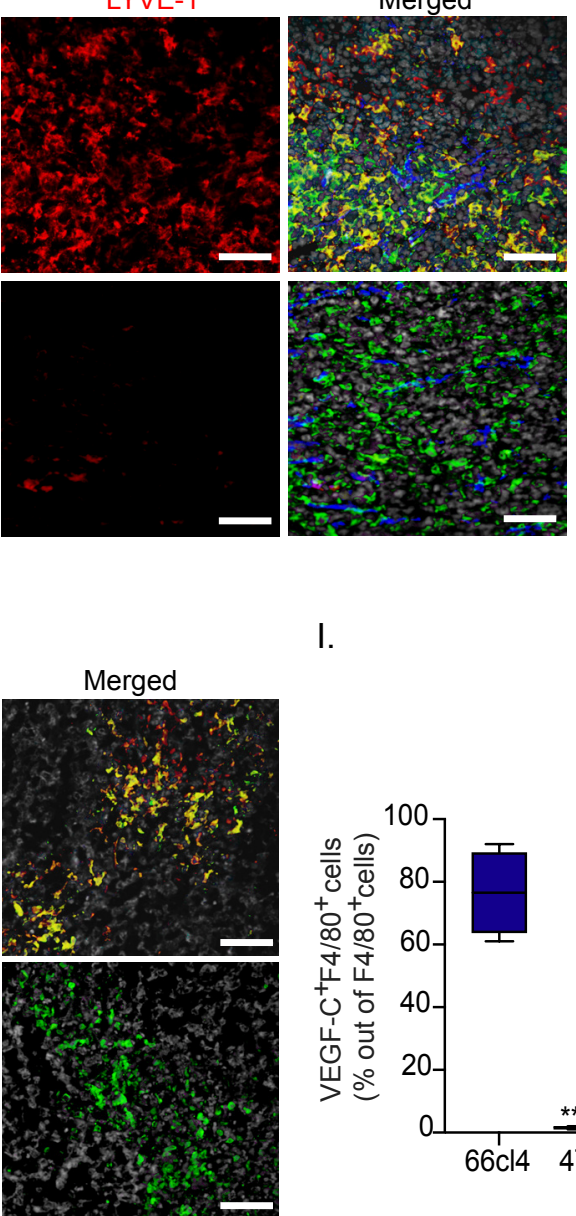

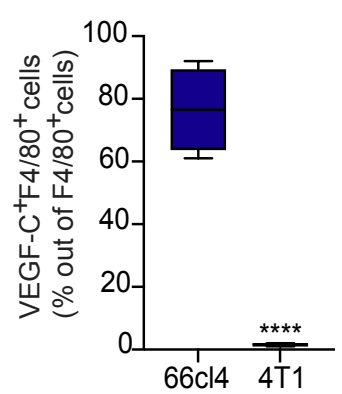

J.

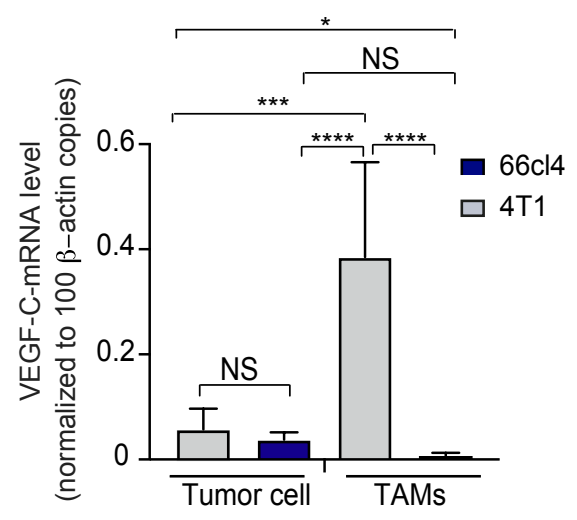


Figure 1. VEGF-C is highly expressed in TAMs derived from $66 \mathrm{cl} 4$ tumors but not 4T1 tumors.

$66 \mathrm{cl} 4$ and $4 \mathrm{~T} 1$ cancer cells were injected into the mammary fat pad of syngeneic mice.

(A) The graph display 66cl4 and 4T1 lung and lymphatic metastatic index (metastatic colonies per gram tumor weight).

B-E, Immunofluorescence analysis by confocal microscopy of 66cl4 and 4T1 tumor sections immunostained for the blood vessel marker CD31 (green), the lymphatic vessel marker VEGFR3 (red) and DAPI (nuclei). Representative images from tumor periphery in (B) and tumor inner region in (D). Morphometric analysis of VEGFR3 ${ }^{+} \mathrm{CD} 31^{-}$lymphatic vessel area (C), and number VEGFR3 ${ }^{+}$CD $31^{+}$blood vessels (E).

F and G, Immunofluorescence analysis by confocal microscopy of $66 \mathrm{cl} 4$ and $4 \mathrm{~T} 1$ tumor sections immunostained for CD31 (blue), the pan-macrophage marker F4/80 (green), the lymphatic vessel marker LYVE-1 (red) and DAPI (nuclei). Representative images in (F), percentage of TAMs expressing LYVE1 in (G).

H-I, Immunofluorescence analysis by confocal microscopy of $66 \mathrm{cl} 4$ and $4 \mathrm{~T} 1$ tumor sections immunostained for F4/80 (green), VEGF-C (red) and DAPI (grey). Representative images in (H) and percentage of TAMs expressing VEGF-C in (I). J, qPCR analysis of VEGF-C in flow cytometry-sorted TAMs or $\mathrm{GFP}^{+}$cancer cells from $4 \mathrm{~T} 1$ and $66 \mathrm{cl} 4$ tumors.

Statistical analyses were performed by Students t-test in (B,C,E,G,I) and by one-way ANOVA in $(\mathrm{J}) ;{ }^{*} p<0.05,{ }^{* *} p<0.01,{ }^{* * *} p<0.001$ and ${ }^{* * * *} p<0.0001 ; n>15$ mice/group; data values are presented as mean + SD. Bars represent $100 \mu \mathrm{m}$ in $(\mathbf{A}, \mathbf{D}, \mathbf{F}, \mathbf{H})$. 
Figure 2 preprint (which was not certified by peer review) is the author/funder. All rights reserved. No reuse allowed without permission.

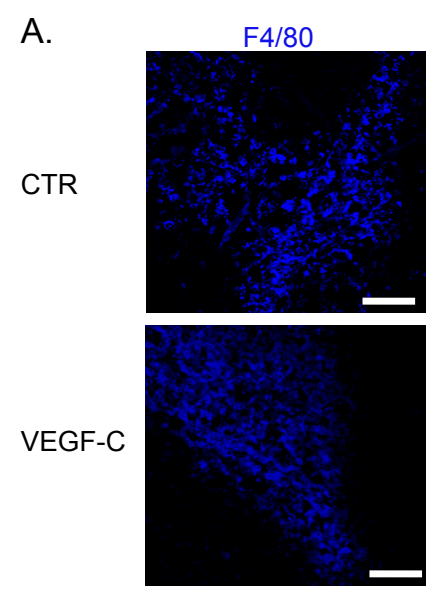

C.

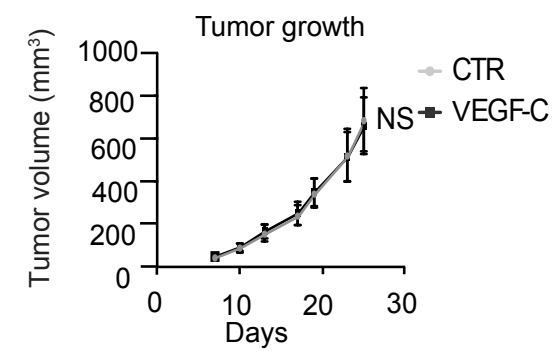

F.

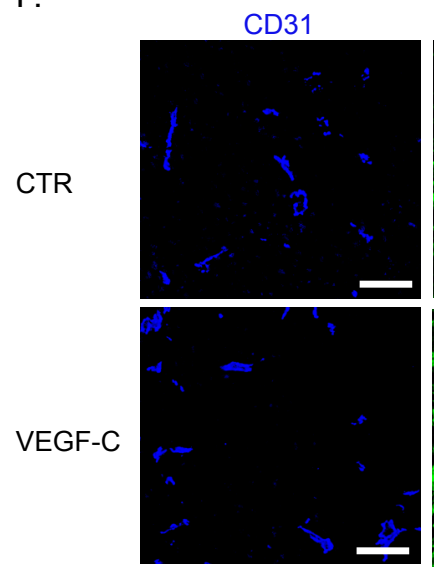

H.
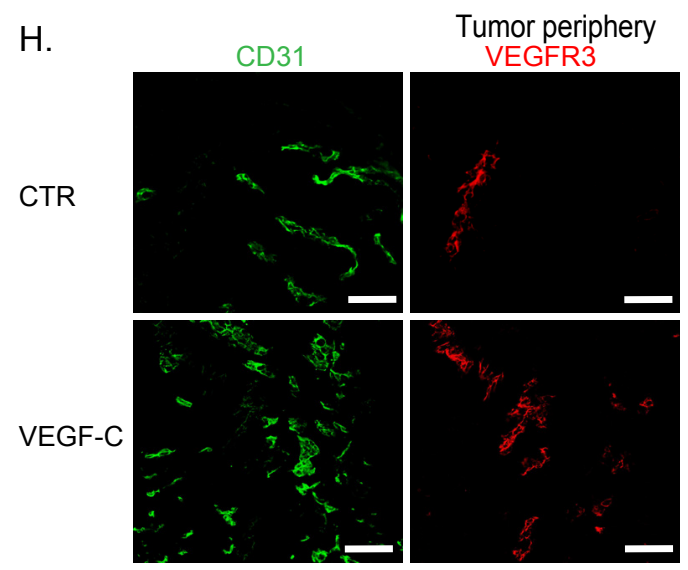

I.

I. VEGFR3 ${ }^{+}$CD31 lymphatic vessels

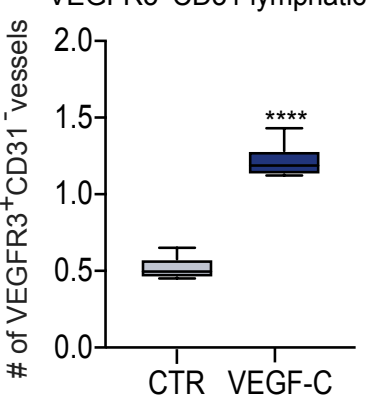

D.

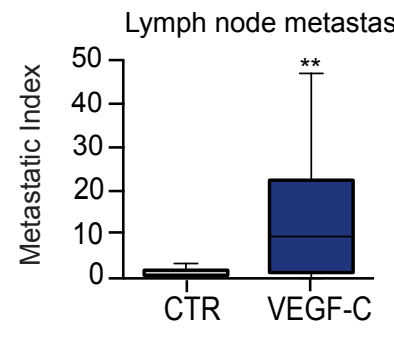

VEGF-C

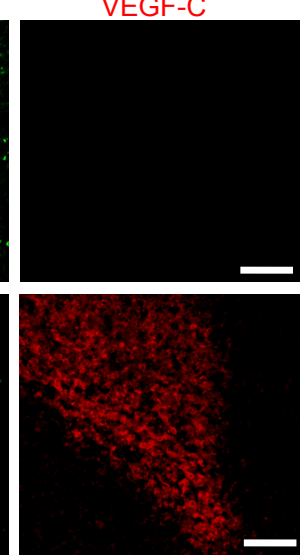

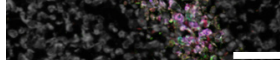

B.
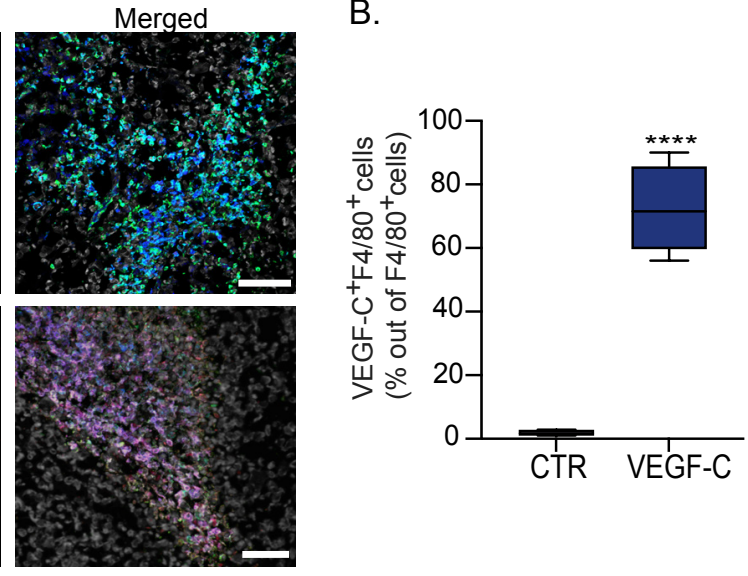

E.

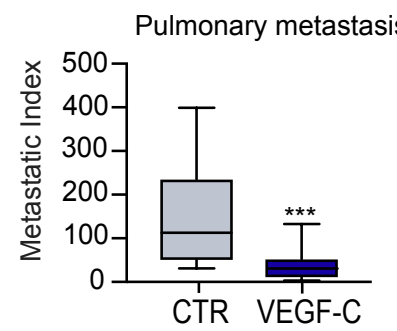

G.

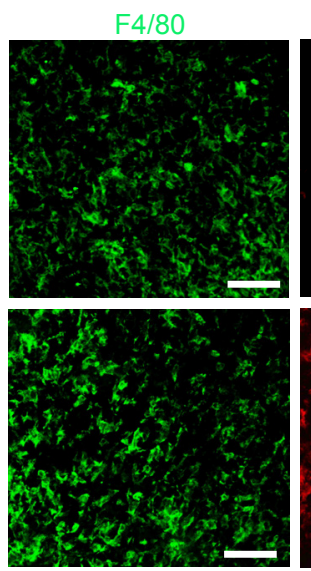

LYVE-1

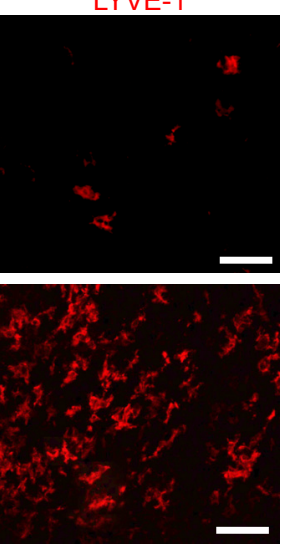

Merged

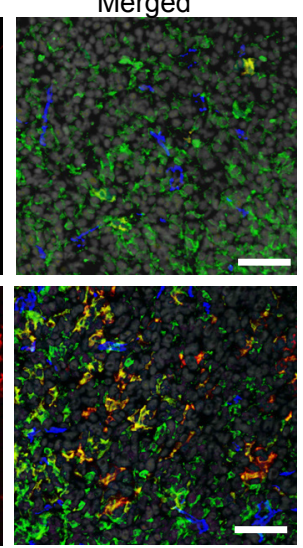

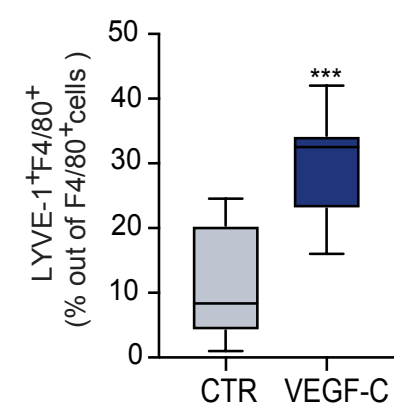

J.
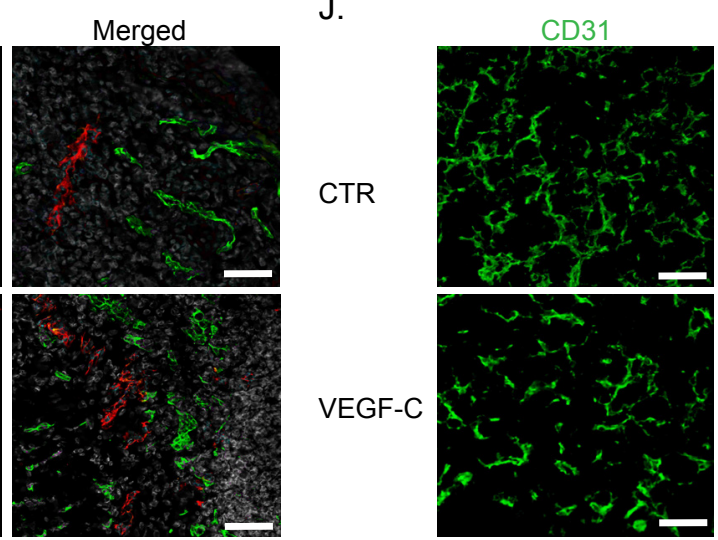

\section{Tumor center}

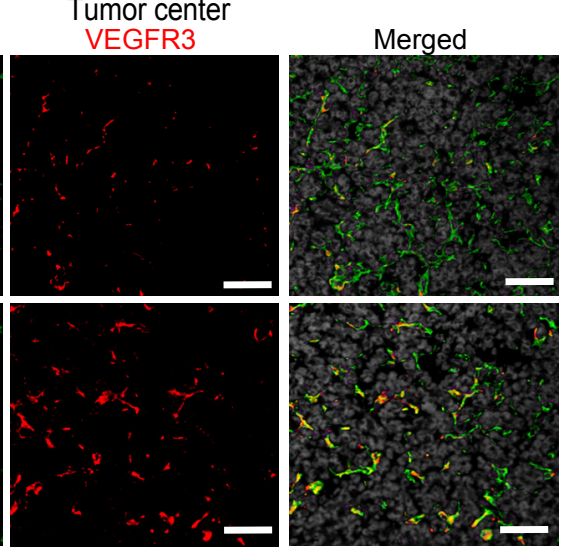

VEGF-C

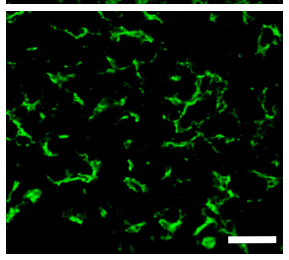

M. CD31+ blood vessel density
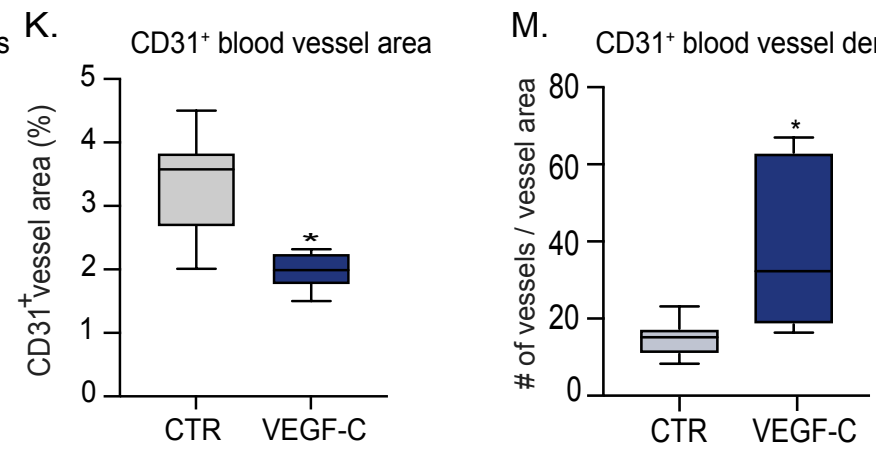


\section{Figure 2. TAM-derived VEGF-C decreases pulmonary metastasis}

4T1 cancer cells were injected into the mammary fat pad of syngeneic chimeric mice harboring haemopoietic cells engineered with VEGF-C or CTR lentiviral vectors.

A-B, Immunofluorescence analysis by confocal microscopy of 4T1 tumor sections immunostained for CD45 (green) F4/80 (blue), VEGF-C (red) and DAPI (grey). Representative images in (A) and percentage of TAMs that express VEGF-C in (B).

C-E, Tumor growth of 4T1 tumors (C), the metastatic index (metastatic colonies per gram tumor weight) in lymph nodes (D) and lungs (E).

F-G, Immunofluorescence analysis by confocal microscopy of tumor sections immunostained for CD31 (blue), F4/80 (green), LYVE1 (red) and DAPI (grey). Representative images in (F) and percentage of LYVE1 ${ }^{+} \mathrm{F} 4 / 80^{+}$TAMs in (G).

H-M, Immunofluorescence analysis by confocal microscopy of 4T1 tumor sections immunostained for CD31 (green), VEGFR3 (red) and DAPI (grey). Representative images in periphery $(\mathbf{H})$ and tumor inner area $(\mathbf{J})$, quantification of VEGFR3 ${ }^{+} \mathrm{CD} 31^{-}$lymphatic vessels in (I), \% of VEGFR3 ${ }^{+} \mathrm{CD} 31^{+}$blood vessels (L), CD $31^{+}$blood vessel area (\%) in (K) and tumor blood vessel density (\# of vessels divided by vessel area per optic field) in (M).

Statistical analyses were performed by Students t-test; ${ }^{*} p<0.05 ;{ }^{* *} p<0.01,{ }^{* * *} p<0.001$ and ${ }^{* * * *} p<0.001 ; n>10$ mice/group. All data values are shown as the mean $+\mathrm{SD}$. Bars represent $100 \mu \mathrm{m}$ in $(\mathbf{A}, \mathbf{F}, \mathbf{H}, \mathbf{J})$. 


\section{Figure 3}

A.

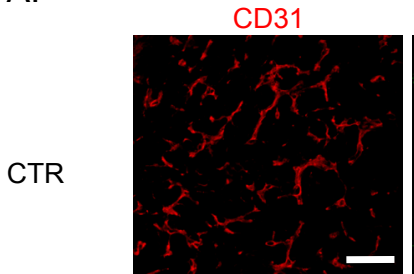

VEGF-C
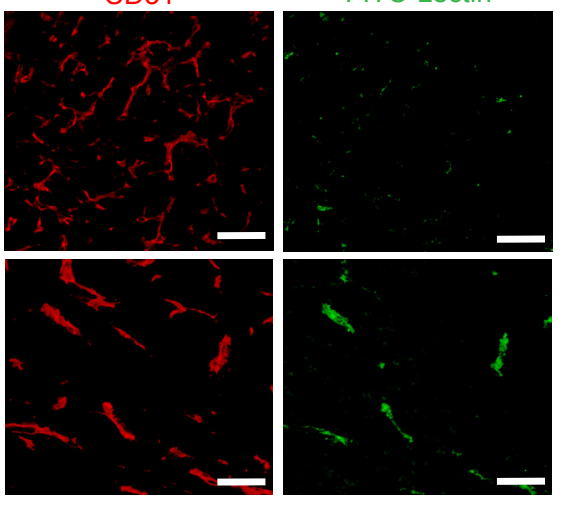

C.

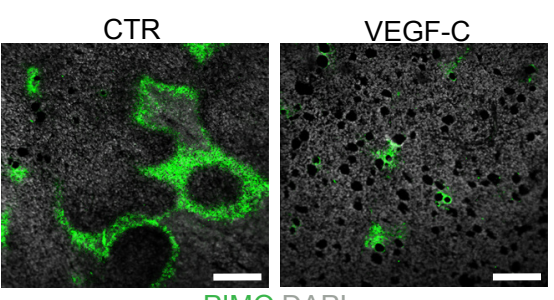

PIMO DAPI

E.

CD31

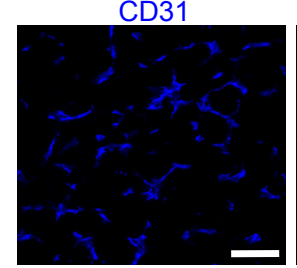

VEGF-C

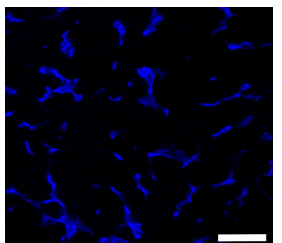

\section{CTR}

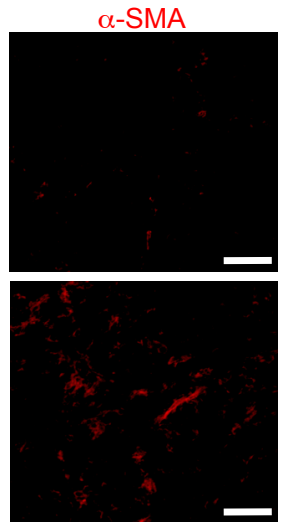

FITC-Lectin

D.

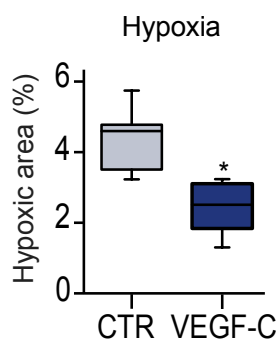

B.
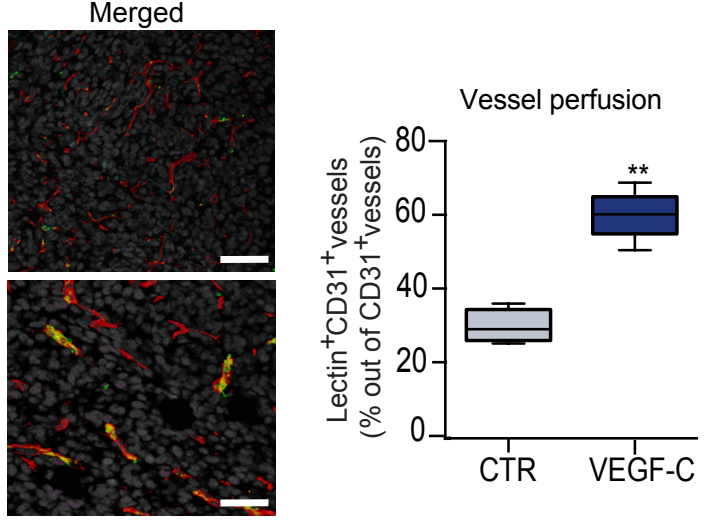

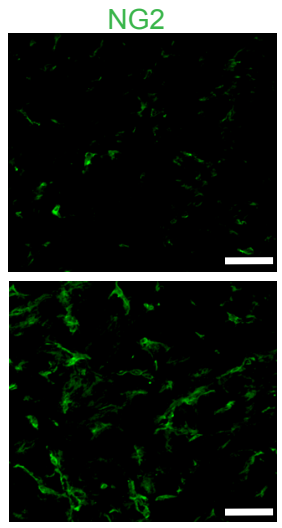

Merged

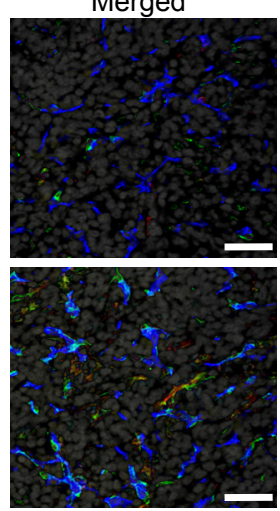

F.

Pericyte covered vessels

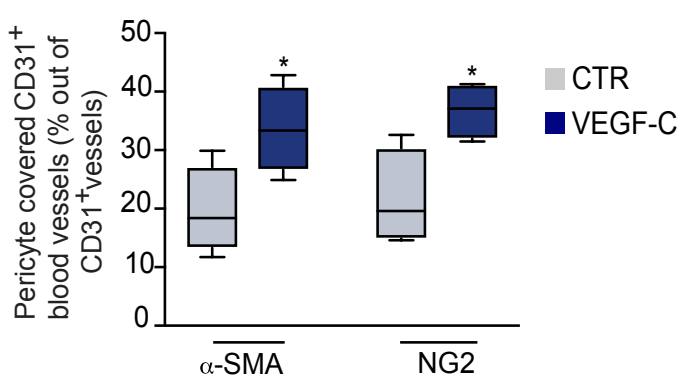


Figure 3. TAM-derived VEGF-C normalizes the tumor vasculature

4T1 cancer cells were injected into the mammary fat pad of syngeneic chimeric mice harboring haemopoietic cells engineered with VEGF-C or CTR lentiviral vectors.

A-F, 4T1 tumors from mice were injected with FITC-lectin i.v. and PIMO i.p. before sacrification. Representative images show immunostaining for CD31 (red) FITC-conjugated lectin (green) and DAPI (grey) in (A), PIMO (hypoxia) and DAPI (grey) in (C). vessel perfusion is quantified in (B) and hypoxic area in (D).

E and F, Representative images of immunofluorescence analysis of 4T1 tumors immunostained for the pericyte markers NG2 (green) and a-SMA (red), CD31 ${ }^{+}$(blue) and DAPI (grey)in (E) and morphometric analysis of $\mathrm{NG}^{+}$or $\alpha-\mathrm{SMA}^{+}$pericyte covered CD31 ${ }^{+}$ blood vessels in $(\mathbf{F})$.

$\mathbf{G}$ and $\mathbf{H}$, Immunofluorescence analysis by confocal microscopy of 4T1 tumors immunostained for CD31 (blue), F4/80 (green), VEGF-C marker (red) and DAPI (grey). Representative images in $(\mathbf{G})$ and quantification of perivascular VEGF-C ${ }^{+} \mathrm{F} 4 / 80^{+}$TAMs in $(\mathbf{H})$.

Statistical analyses were performed by Students t-test; ${ }^{*} p<0.05$, and ${ }^{* *} p<0.01 ; n>10$ mice/group. All data values are shown as the mean + SD. Bars represent $100 \mu \mathrm{m}$ in $(\mathbf{A}, \mathbf{C}, \mathbf{E})$. 
Figure 4 preprint (which was not certified by peer review) is the author/funder. All rights reserved. No reuse allowed without permission.

A.

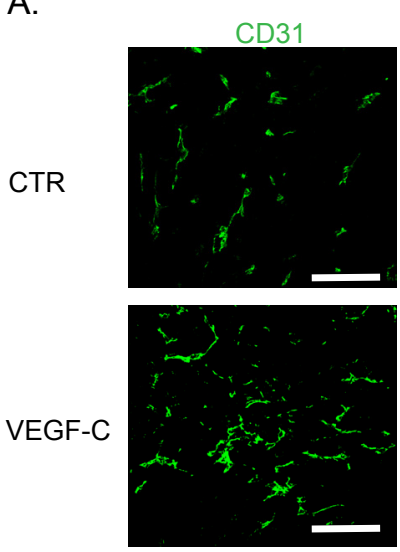

C.

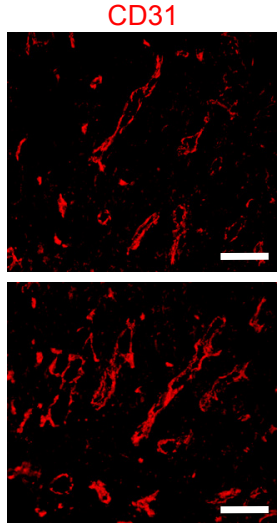

E.

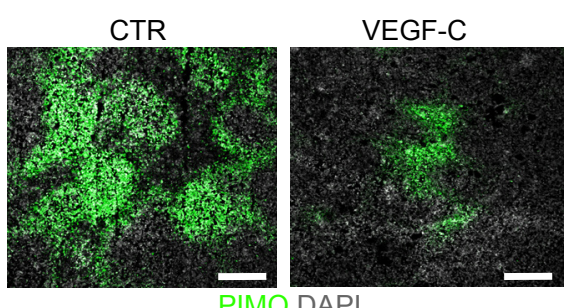

G.

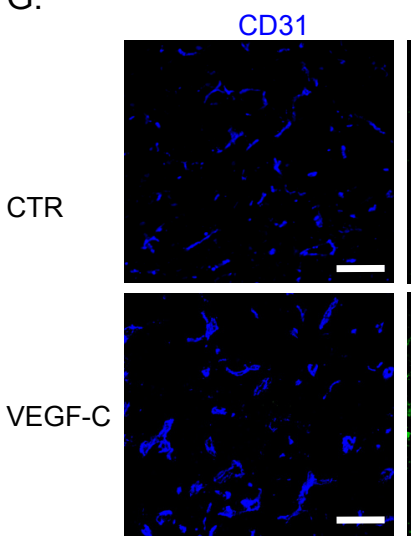

I.

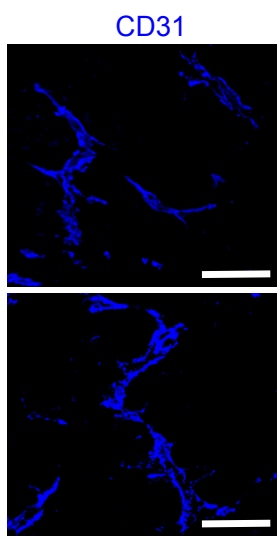

Tumor center
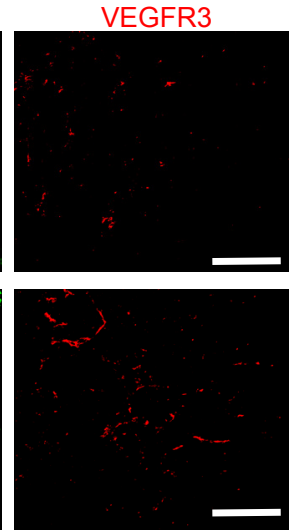

FITC-lectin
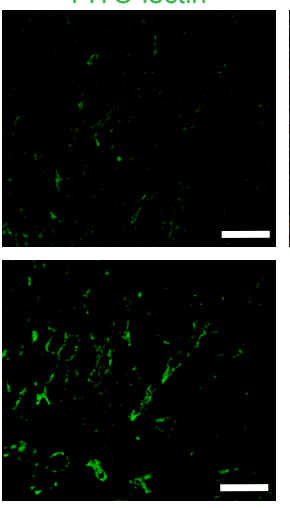

-

NG2

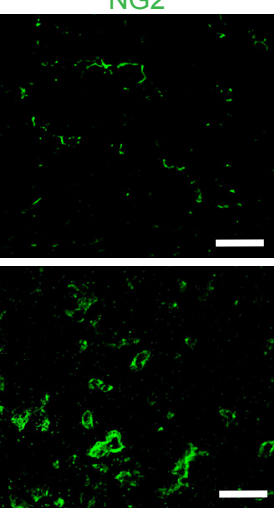

F4/80

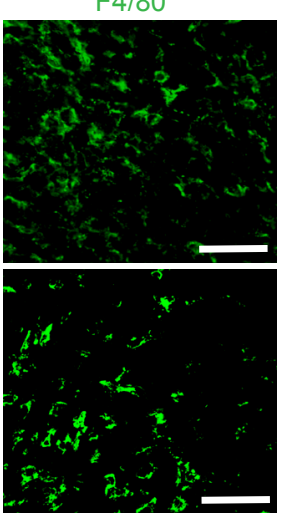

\section{CTR}

VEGF-C
Merged

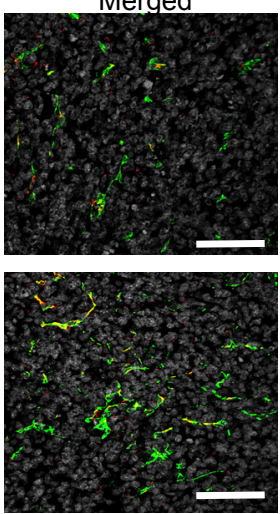

Merged

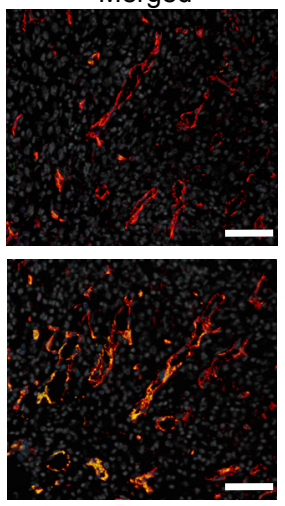

F. Hypoxia

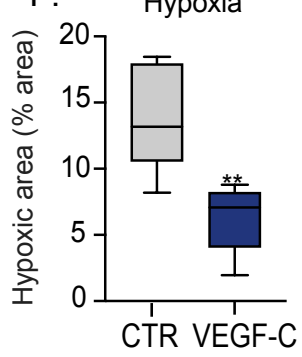

B.

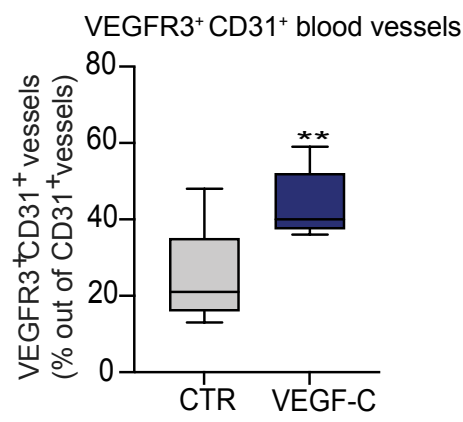

D.

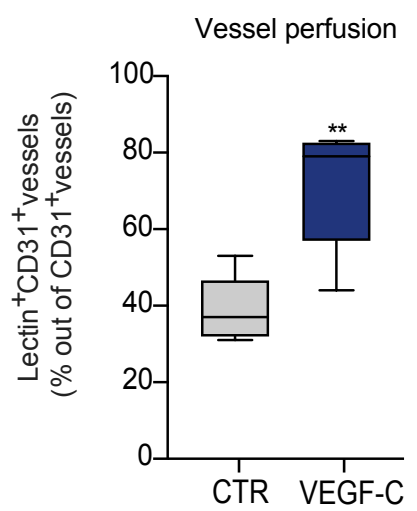

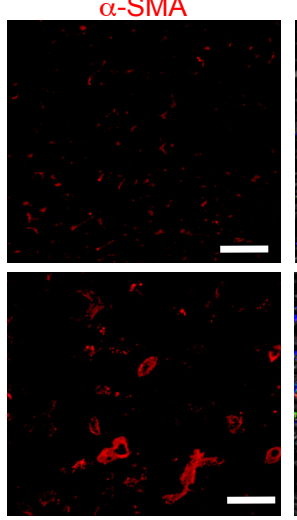

MENA

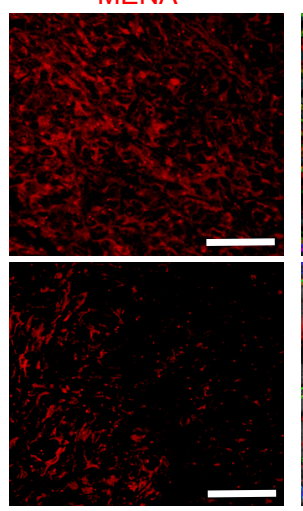

Merged

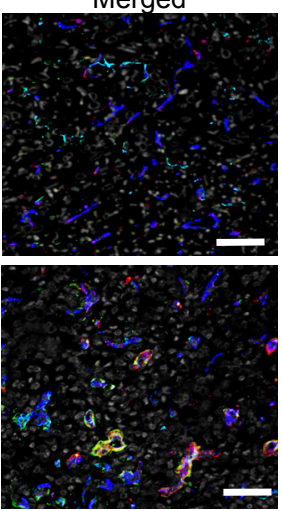

Merged

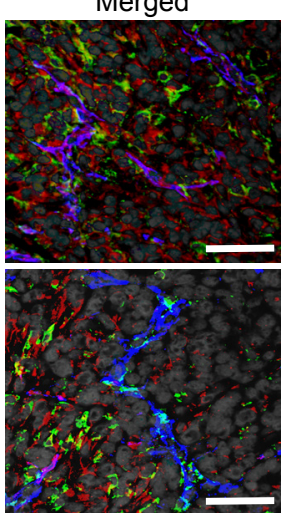

H.

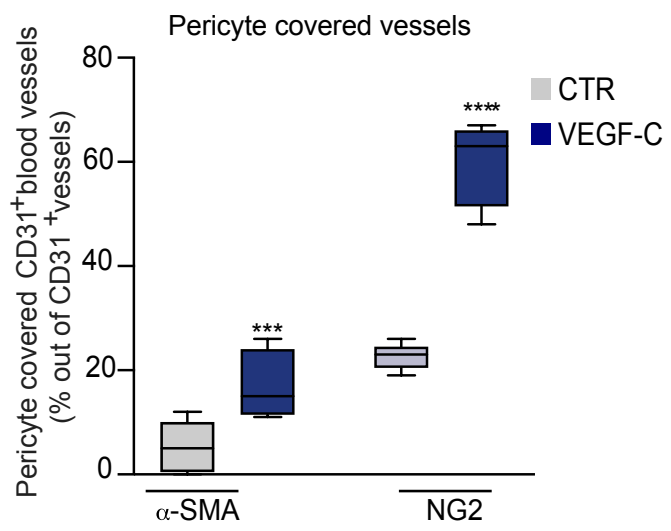

J.

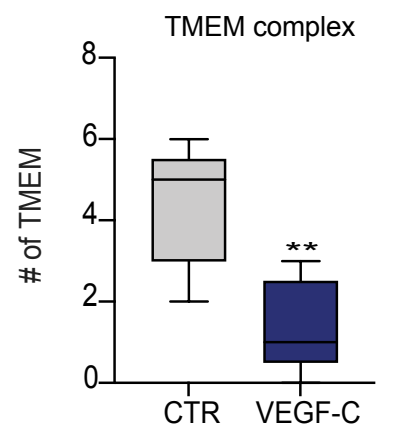




\section{Figure 4. TAM-derived VEGF-C induces vascular normalization}

4T1 cancer cells were co-mingled in matrigel with BMDMs previously transduced with CTR or VEGF-C LVs and injected subcutaneously into syngeneic mice.

A-B, Representative images shows the tumor inner area of CD31 (green), VEGFR3 (red) and DAPI (grey). Proportion VEGFR3 ${ }^{+}$CD $31^{+}$blood vessels, quantified in (B).

C-F, CTR and VEGF-C tumor bearing mice were injected with FITC-conjugated lectin and PIMO before sacrification. Tumor slides were immunostained for CD31 (red), FITC-conjugated lectin (green) and DAPI (grey) in (C) or for PIMO (green) and DAPI (grey) in (E). Morphometric analysis of proportion of perfused vessels is shown in (D) and hypoxic area in (F).

G-H, Representative images for NG2 (green), a-SMA (red), CD31 (blue) and DAPI (grey) in (G). The graph displays morphometric analysis of pericyte covered blood vessels in $(\mathbf{H})$.

I-J Immunostaining for CD31 (blue), F4/80 (green), MENA (red) and DAPI (grey) in (E). the number of TMEM complexes normalized to the number of vessels are quantified in $(\mathbf{J})$.

Statistical analyses were performed by Students t-test; ${ }^{* *} p<0.01$ and ${ }^{* * *} p<0.001 ; n>10$ mice/group. All data values are shown as the mean + SD. Bars represent $100 \mu \mathrm{m}$ in (A,C,E, G and I). 
Figure 5 preprint (which was not certified by peer review) is the author/funder. All rights reserved. No reuse allowed without permission.

A.

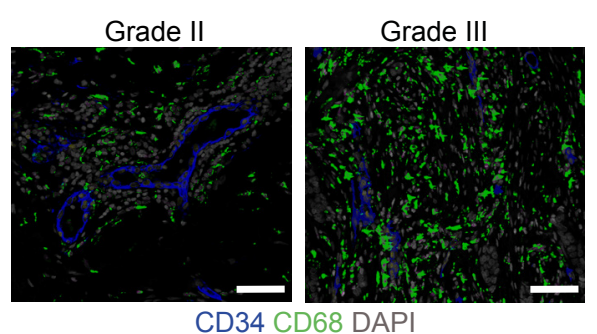

D.

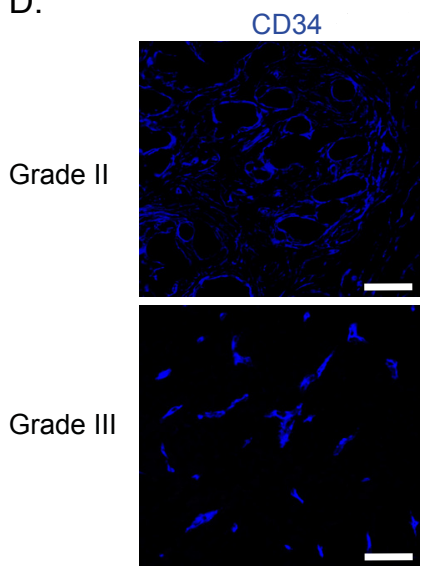

F.

H.

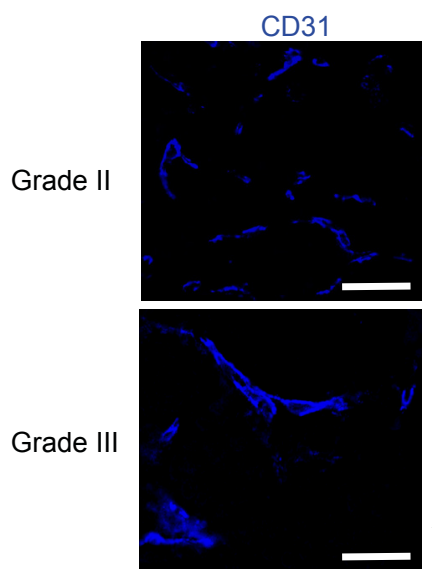

J.

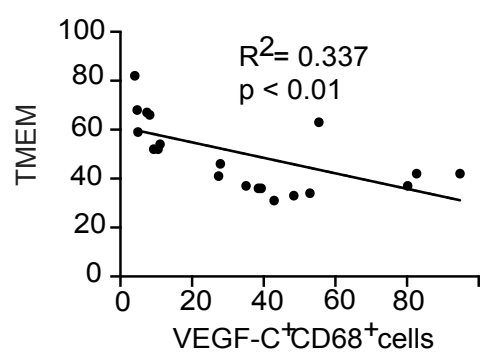

Grade II

Grade III
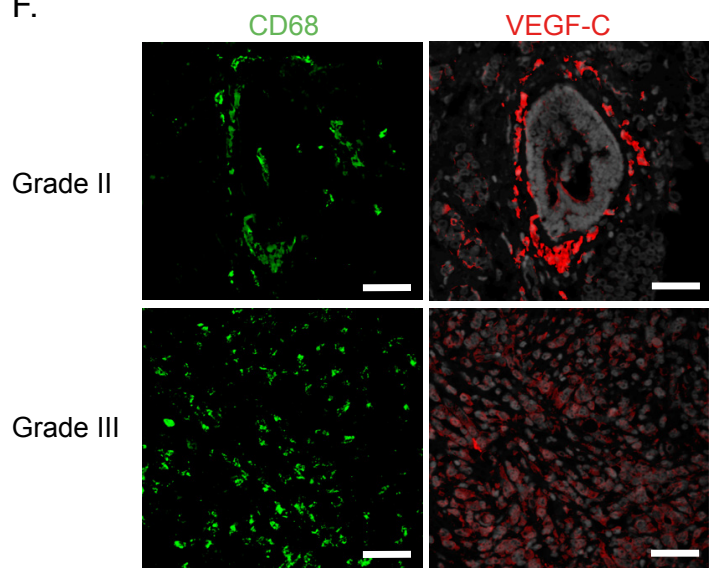

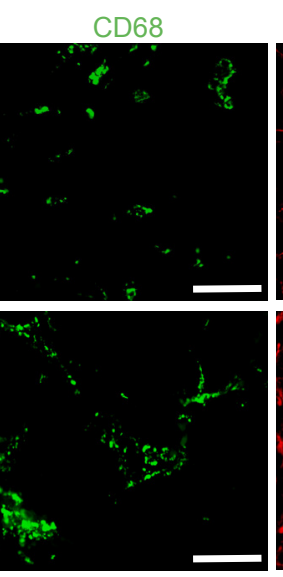

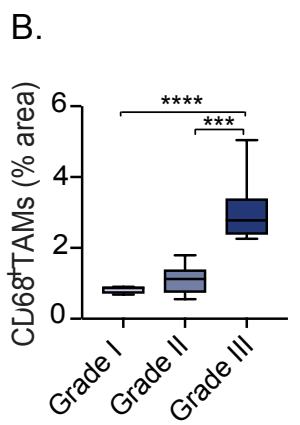

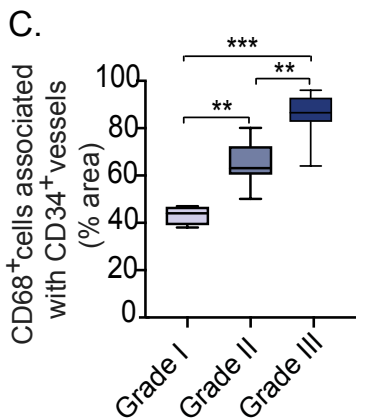

VEGF-C

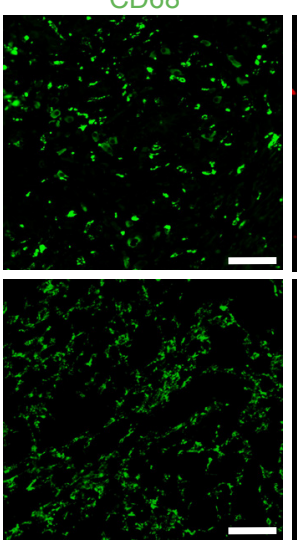

Merged

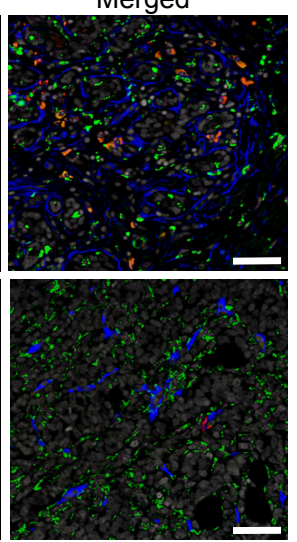

E.

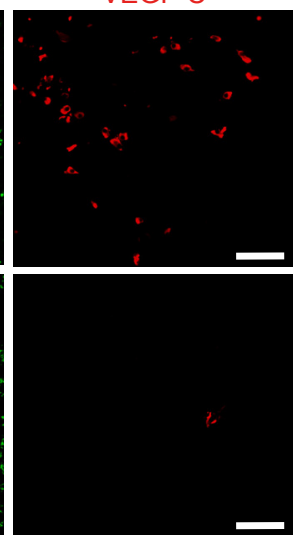

G.
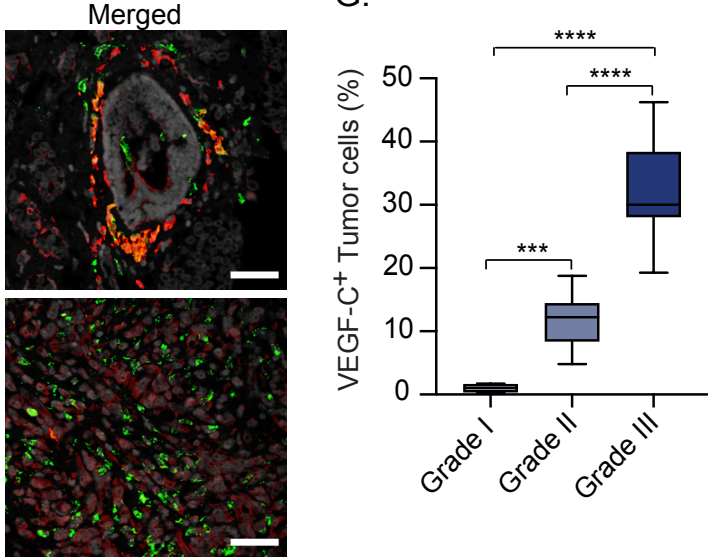

I.

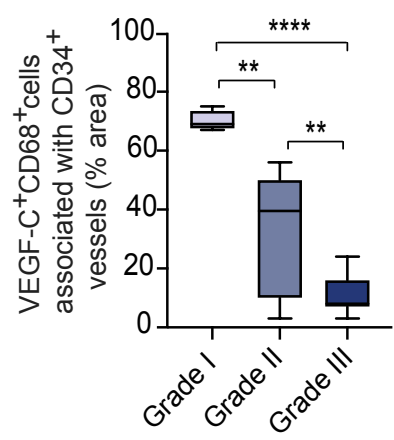

MENA
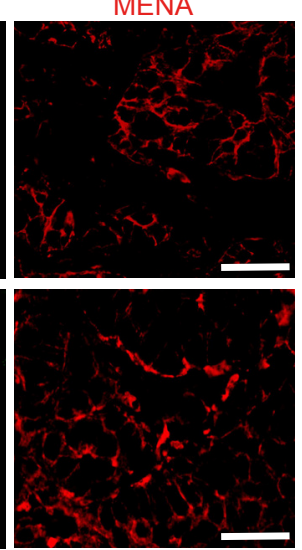

K.
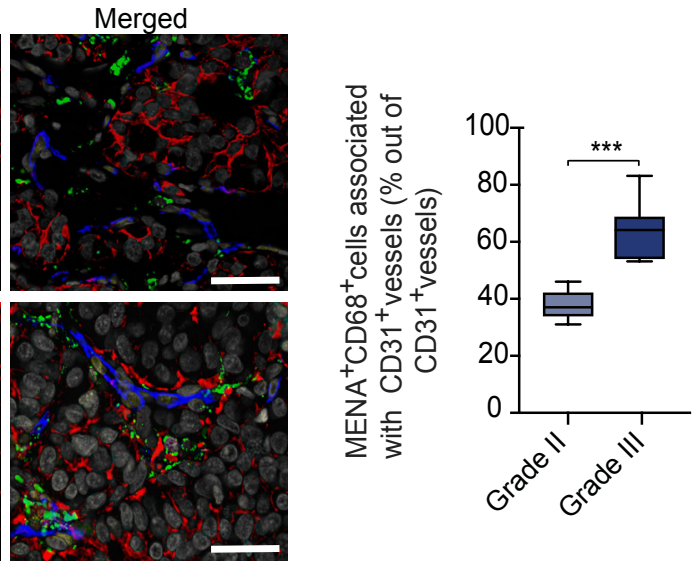
Figure 5. Perivascular TAM-derived VEGF-C inversely correlates to the TMEM complex and grade of malignancy in human breast cancer

A-C, Immunofluorescence analysis by confocal microscopy of human $\mathrm{ER}^{+}$breast tumors immunostained for CD31 (blue); pan-macrophage marker CD68 (green) and nuclei (DAPI; grey). Representative images in (A), quantitative analysis of CD68 ${ }^{+}$TAMs in (B) and CD68 ${ }^{+}$ perivascular TAMs in (C).

D and E, Immunofluorescence analysis by confocal microscopy of human ER ${ }^{+}$breast tumors immunostained for CD68 (green), CD31 (blue) and VEGF-C (red). Representative images in (D) and quantitative analysis of VEGF-C positive perivascular TAMs in (E).

F and G, Immunofluorescence analysis by confocal microscopy of human breast tumors immunostained for CD68 (green), and VEGF-C (red). Representative images in (F) and quantitative analysis of VEGF-C positive cancer cells in (G).

H-K, Immunofluorescence analysis by confocal microscopy of human breast tumors immunostained for CD68 (green), CD31 (blue), MENA (red) and (DAPI). Representative images in $\mathbf{( H )}$, quantitative analysis of TMEM complexes ( a MENA positive cancer cell, a CD68 positive TAM associated with a CD31 ${ }^{+}$endothelial cell) in (I), Pearson's correlation ( $\mathrm{R}^{2 ;}$ $\left.{ }^{* *} p<0.01\right)$ between the percentage of TMEM complexes and VEGF-C-expressing TAMs $(\mathbf{J})$ and the percentage of TMEM complexes and VEGF-C-expressing cancer cells (K). Statistical analyses were performed by one-way ANOVA in (B,C,E,G) and by Students t-test in (I); ${ }^{* *} p<0.01{ }^{* * *} p<0.001,{ }^{* * *} p<0.0001, n=4$ for grade $\mathrm{I}, \mathrm{n}=11$ for grade II and 9 for grade III -. Data are presented as the mean + SD. Bars represent $100 \mu \mathrm{m}(\mathbf{A}, \mathbf{D}, \mathbf{F})$ and $50 \mu \mathrm{m}$ in $(\mathbf{H})$. 
Figure 6

A.

Tumor

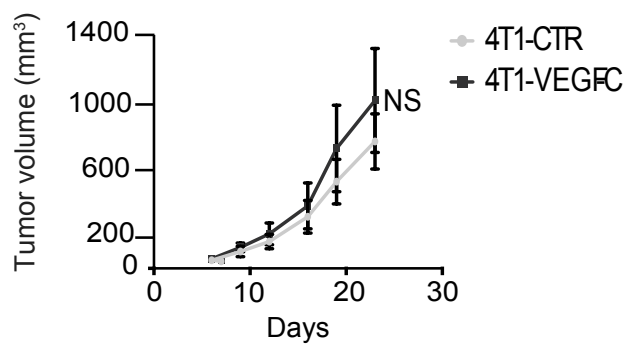

D.

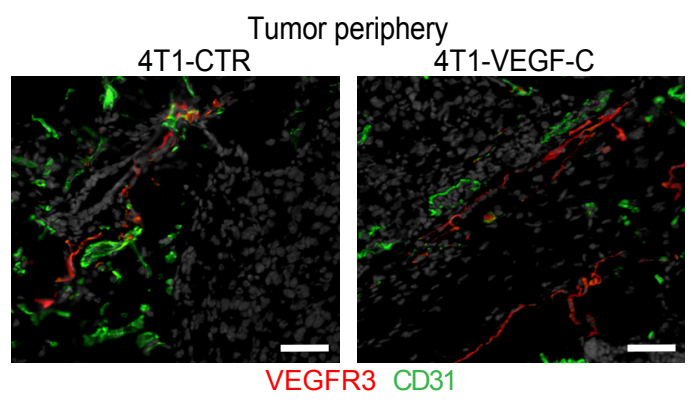

F.

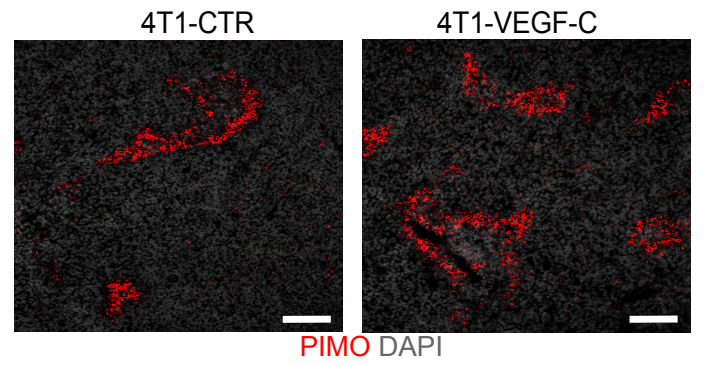

H.

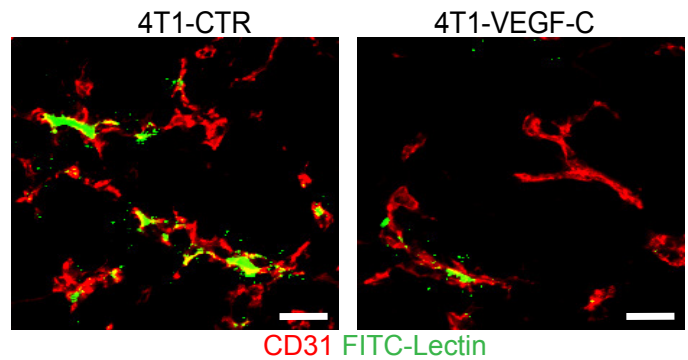

J.

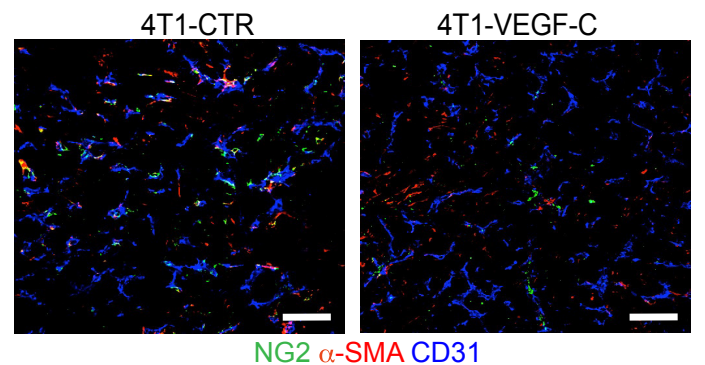

M.

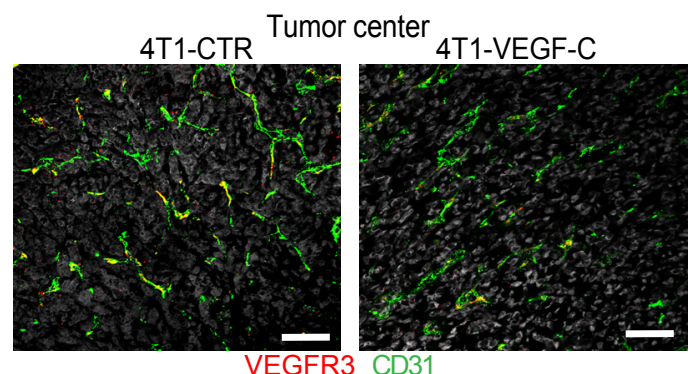

Lymph node metastasis
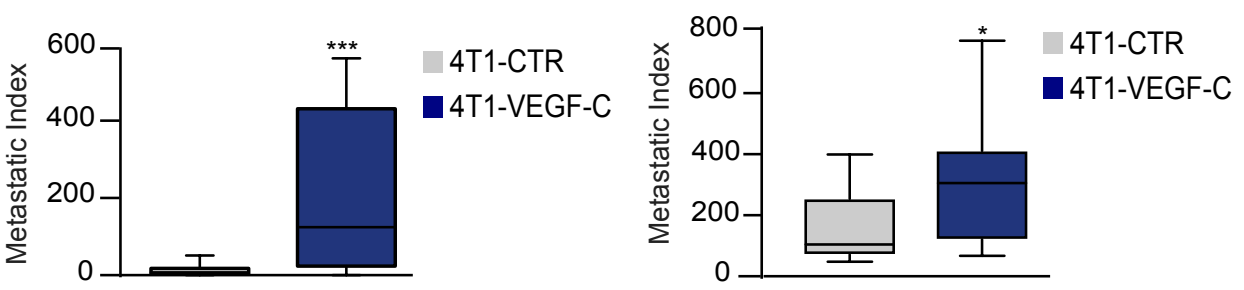

E.

VEGFR3 ${ }^{+} \mathrm{CD} 31^{-}$lymphatic vessels

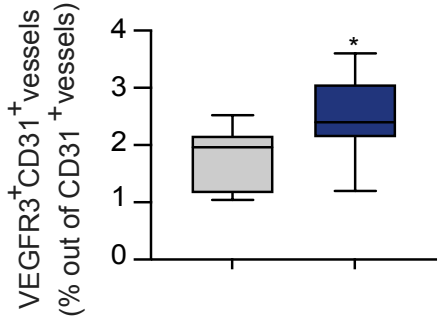

4T1-CTR

4T1-VEGF-C

G.

Hypoxia

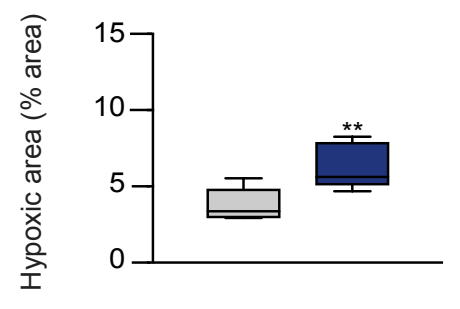

4T1-CTR

4T1-VEGF-C

I.

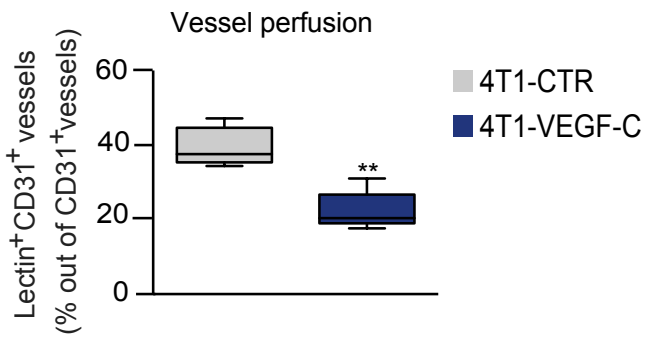

K.

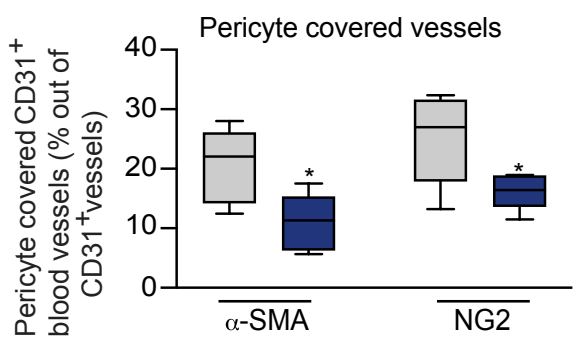

N. VEGFR $3^{+} \mathrm{CD} 31^{+}$blood vessels

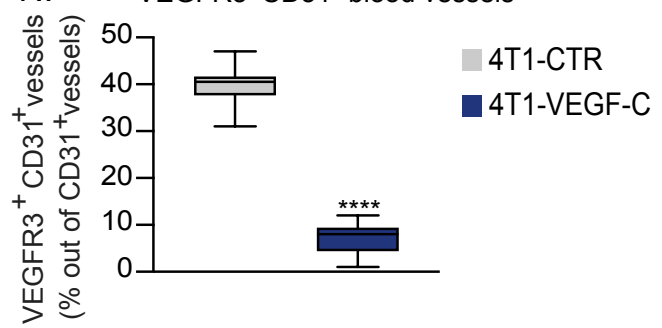

4T1-CTR

4T1-VEGF-C 
Figure 6. Cancer cell-derived VEGF-C enhances vascular abnormality coinciding with increased proximal and distant metastases

4T1 cancer cells transduced with CTR or VEGF-C vectors were inoculated into the fat pad of syngeneic mice.

A-C, Graphs display tumor growth in (A) metastatic index for lymph nodes in (B) and lungs in (C).

D-K, Immunofluorescence analysis by confocal microscopy of 4T1 tumors immunostained for lymphatic vessels CD31 (red) and VEGFR3 (green). Representative images in (D) and morphometric analysis of VEGFR3 ${ }^{+}$Iymphatic vessel area in (E).

F and I, 4T1 tumors bearing mice were injected with PIMO and 2000 kDa-dextran before scarification. Representative images show immunostaining for PIMO (red) and DAPI (grey) in (F) and perfused vessels in (H). Graphs display quantification of hypoxic area in (E). and proportion of vessels containing $2000 \mathrm{kDa}$ dextran in (I).

J -K, Immunofluorescence analysis by confocal microscopy of 4T1 tumors immunostained for NG2 (green), a-SMA (red), CD31 (blue) and DAPI (grey). Representative images in (H) and quantification of pericyte covered vessels in (I).

M-N, Representative images shows the tumor inner area of CD31 (green), VEGFR3 (red) and DAPI (grey). Proportion VEGFR3 ${ }^{+}$CD $31^{+}$blood vessels, quantified in (B).

Statistical analysis was performed by Students t-test; ${ }^{*} p<0.05 ;{ }^{* *} p<0.01{ }^{* * *} p<0.001$, ${ }^{* * * *} p<0.0001$. Data are presented as the mean + SD. Bars represent $100 \mu \mathrm{m}(\mathbf{D}, \mathbf{F}, \mathbf{H}, \mathbf{J})$. 